\title{
Corner-corrected diagonal-norm summation-by-parts operators for the first derivative with increased order of accuracy
}

DOI:

10.1016/j.jcp.2016.10.051

\section{Document Version}

Accepted author manuscript

Link to publication record in Manchester Research Explorer

Citation for published version (APA):

Del Rey Fernández, D. C., Boom, P. D., \& Zingg, D. W. (2017). Corner-corrected diagonal-norm summation-byparts operators for the first derivative with increased order of accuracy. Journal of Computational Physics, 330, 902-923. https://doi.org/10.1016/j.jcp.2016.10.051

\section{Published in:}

Journal of Computational Physics

\section{Citing this paper}

Please note that where the full-text provided on Manchester Research Explorer is the Author Accepted Manuscript or Proof version this may differ from the final Published version. If citing, it is advised that you check and use the publisher's definitive version.

\section{General rights}

Copyright and moral rights for the publications made accessible in the Research Explorer are retained by the authors and/or other copyright owners and it is a condition of accessing publications that users recognise and abide by the legal requirements associated with these rights.

\section{Takedown policy}

If you believe that this document breaches copyright please refer to the University of Manchester's Takedown Procedures [http://man.ac.uk/04Y6Bo] or contact uml.scholarlycommunications@manchester.ac.uk providing relevant details, so we can investigate your claim.

\section{OPEN ACCESS}




\title{
Corner-Corrected Diagonal-Norm Summation-By-Parts Operators for the First Derivative With Increased Order of Accuracy
}

\author{
David C. Del Rey Fernández ${ }^{\mathrm{a}, *}$, Pieter D. Boomª,**, David W. Zingga, \\ ${ }^{a}$ Institute for Aerospace Studies, University of Toronto, Toronto, Ontario, M2H 5T6, \\ Canada
}

\begin{abstract}
Combined with simultaneous approximation terms, summation-by-parts (SBP) operators offer a versatile and efficient methodology that leads to consistent, conservative, and provably stable discretizations. However, diagonal-norm operators with a repeating interior-point operator that have thus far been constructed suffer from a loss of accuracy. While on the interior, these operators are of degree $2 p$, at a number of nodes near the boundaries, they are of degree $p$, and therefore of global degree $p$ - meaning the highest degree monomial for which the operators are exact at all nodes. This implies that for hyperbolic problems and operators of degree greater than unity they lead to solutions with a global order of accuracy lower than the degree of the interior-point operator. In this paper, we develop a procedure to construct diagonal-norm first-derivative SBP operators that are of degree $2 p$ at all nodes and therefore can lead to solutions of hyperbolic problems of order $2 p+1$. This is accomplished by adding nonzero entries in the upper-right and lower-left corners of SBP operator matrices with a repeating interior-point operator. This modification necessitates treating these new operators as elements, where mesh refinement is accomplished by increasing the number of elements in the mesh rather than increasing the number of nodes. The significant improvements in accuracy of this new family, for the same repeating interior-point operator, are demonstrated in the context of the linear
\end{abstract}

\footnotetext{
*Corresponding author, Post-doctoral fellow: dcdelrey@gmail.com

**Post-doctoral fellow: pieter.boom@mail.utoronto.ca

Professor, Institute for Aerospace Studies, J. Armand Bombardier Foundation Chair in Aerospace Flight. AIAA Associate Fellow.
} 
convection equation.

Keywords: First derivative, summation-by-parts, energy method, quadrature, simultaneous approximation terms, finite-difference methods, discontinuous-Galerkin methods, continuous-Galerkin methods, partial-differential equations 2000 MSC: 65D25, MSC 65D30 2000 MSC: 6532

\section{Introduction}

Nodal methods with the summation-by-parts property (SBP) $[1,2,3,4,5]$ have a number of attractive properties. When combined with simultaneous approximation terms (SATs) [6, 7, 8, 9] for the weak imposition of bound5 ary conditions and inter-element coupling, SBP operators lead to consistent, conservative and provably stable discretizations of partial differential equations (PDEs). This results from the fact that SBP operators are mimetic of integration by parts and are amenable to the application of the energy method for proving stability. Recently it has been shown that such operators can be developed for structured or unstructured meshes [10], and mesh refinement can be accomplished using a traditional finite-difference (FD), a discontinuous-element, or a continuous-element approach [10].

Although an SBP operator, $\mathrm{D}_{\xi}=\mathrm{H}_{\xi}^{-1} \mathrm{Q}_{\xi}$, approximating the first derivative, with respect to the curvilinear coordinate $\xi$, can be constructed to have a norm matrix ${ }^{1} \mathrm{H}_{\xi}$ that is dense, in this paper, only diagonal-norm SBP operators are considered - examination of dense-norm operators is deferred to a later paper. Classical FD-SBP methods have a long history of development and many of the issues related to applying such methods to real-world problems to a large extend have been resolved. However, classical diagonal-

20 norm FD-SBP operators suffer from a loss of accuracy at and near boundary nodes. While these operators are of degree $2 p$ at interior nodes, that is the highest power monomial for which they are exact, a number of point operators at and near boundary nodes are of degree $p$; therefore, the global degree of these operators is $p$, by which we mean the maximum degree monomial for which the derivative operator is exact at all nodes. The error properties of classical FD-SBP operators can be improved by constructing operators

\footnotetext{
${ }^{1}$ The norm matrix is synonymous with the mass matrix in finite-element methods.
} 
on nodal distributions that are nonuniform on a finite set of nodes near the boundaries $[11,12]$. However, these operators are still of degree $p$ at a number of boundary nodes and are therefore of global degree $p$.

Until recently, the diagonal-norm SBP operators with a repeating interiorpoint operator on a uniform nodal distribution reported in the literature have been of global degree less than or equal to five. Albin and Klarmann [13], have subsequently demonstrated that such operators of global degree $>5$ can be constructed. However, the resulting operators require increasingly larger numbers of lower degree-boundary point operators and require careful optimization to be useful (see also [14]). Their is considerable interest in developing new efficient high-order SBP schemes that can be utilized within existing production level codes based on classical FD-SBP operators.

The objective of this paper is to improve the efficiency of tensor-product SBP methods that have operators with a repeating interior-point operator. Such operators allow great flexibility in the specification of the degrees of freedom within an element independent of the global degree of the scheme; this results in numerical methods that retain the same numerical characteristics, for example sparsity and global degree, regardless of how many nodes nodal discontinuous Galerkin method, have fundamentally different characteristics as the number of nodes within the element is altered; this results because there is a one-to-one coupling between the number of nodes in the element and the global degree of the operator. The ability to specify the allel schemes that are architecture optimized; it allows the specification of the computational intensity of the operator and the number of interfaces for a specified nodal density, thereby reducing communication overhead and maximizing latency hiding.

In this paper, we propose a class of SBP operators that have the same degree as the interior-point operator - a preliminary study of this class of operators was presented in Ref. [1]. Our goal is to develop an approach that leads to general solutions for this class of operators and construct several families of these operators on nodal distributions in computational space. We examine operators constructed on uniform nodal distributions which can be used immediately within the context of codes based on classical FD SBP operators. In addition, we take advantage of the generalization in Ref. [3] and construct operators on the hybrid-Gauss-trapezoidal-Lobatto and hybrid-Gausstrapezoidal nodal distributions that have a number of unequally spaced nodes 
near the boundaries, and result in smaller truncation error at boundary-point operators. Finally, we demonstrate the properties of the new families of operators by solving the two-dimensional linear-convection equation on Cartesian and curved meshes.

The paper is organized as follows: in Section 2, the notation used in the paper is delineated. SBP operators for the first derivative and the three means of implementing them are reviewed in Sections 3 and 4, respectively. The development of the new family of SBP operators and their construction is detailed in Section 5, while the resultant operators are characterized in the context of the linear convection equation in Section 6. Finally, conclusions are drawn in Section 7.

\section{Notation and definitions}

The conventions used in this paper are a combination of those given in Refs. [1, 3, 12, 15], and readers familiar with the conventions may wish to skip to Section 3. We are interested in tensor-product methods that can be applied on curvilinear coordinates $(\xi, \eta)$. Operators for multi-dimensional discretizations are constructed from tensor-products of one-dimensional operators. Thus, for simplicity, much of the presentation in this paper concentrates on one dimensional operators and the notation presented in this section reflects this choice. Vectors are denoted with small bold letters, for example, $\boldsymbol{u}=\left[\mathcal{U}\left(\xi_{1}\right), \ldots, \mathcal{U}\left(\xi_{N}\right)\right]^{\mathrm{T}}$, while matrices are presented using capital letters with sans-serif font, for example, M. Capital letters with a script type are used to denote continuous functions on a specified domain $\xi \in\left[\xi_{\mathrm{L}}, \xi_{\mathrm{R}}\right]$. As an example, $\mathcal{U}(\xi) \in C^{\infty}\left[\xi_{\mathrm{L}}, \xi_{\mathrm{R}}\right]$ denotes an infinitely differentiable function on the domain $\xi \in\left[\xi_{\mathrm{L}}, \xi_{\mathrm{R}}\right]$. Lowercase bold font is used to denote the restriction of such functions onto a grid; for example, the restriction of $\mathcal{U}$ onto the computational grid $\boldsymbol{\xi}$ is given by

$$
\boldsymbol{u}=\left[\mathcal{U}\left(\xi_{1}\right), \ldots, \mathcal{U}\left(\xi_{N}\right)\right]^{\mathrm{T}} .
$$

Vectors with a subscript $h$, for example, $\boldsymbol{u}_{h} \in \mathbb{R}^{N \times 1}$, represents the solution to a system of discrete or semi-discrete equations.

Throughout this paper, the restriction of monomials is represented by $\boldsymbol{\xi}^{k}=\left[\xi_{1}^{k}, \ldots, \xi_{N}^{k}\right]^{\mathrm{T}}$, with the convention that $\boldsymbol{\xi}^{k}=\mathbf{0}$ if $k<0$. We discuss the global degree of SBP operators, $p$, by which we mean the degree of the highest monomial for which they are exact, as well as the degree at specific nodes. 
As discussing the degree of operators is not typical in the FD community, we clarify its meaning here. The approximation of the derivative has a leading truncation error term for each node, proportional to some power of $\Delta x$, the mesh spacing. For the first derivative, the order and the degree of the point operators is the same. More generally, the relation between the two for the $m^{\text {th }}$ derivative is

$$
\text { degree }=\text { order }+m-1 .
$$

Henceforth we will refer to the order of the solution error and discuss the accuracy of the operators exclusively in terms of degree.

\section{Summation-by-parts operators}

SBP operators are constructed to discretely preserve the integration-byparts property of first and higher derivative $[11,12,16,17]$ such that the energy method can be applied to prove the stability of the semi-discrete or fully-discrete system of equations.

For the reader unfamiliar with the SBP concept we present a very brief overview; those interested in further details are referred to the texts $[18,19]$ and the two review papers $[1,2]$.

Consider the linear convection equation with unit wave speed in curvilinear coordinate $\xi=\xi(x)$,

$$
\frac{\partial \mathcal{J}^{-1} \mathcal{U}}{\partial t}=-\frac{\partial \mathcal{U}}{\partial \xi}, \quad \xi \in\left[\xi_{\mathrm{L}}, \xi_{\mathrm{R}}\right], \quad t \geq 0
$$

where $\mathcal{J}$ is the Jacobian of the transformation. The initial condition and a Dirichlet boundary condition are given by

$$
\mathcal{U}(\xi, 0)=\mathcal{F}(\xi), \quad \mathcal{U}\left(\xi_{\mathrm{L}}, t\right)=\mathcal{G}_{L}(t)
$$

respectively. The stability of the problem defined by (1) and (2) can be determined by using the energy method. The first step is to multiply (1) by the solution and integrate in curvilinear space, resulting in

$$
\int_{\xi_{\mathrm{L}}}^{\xi_{\mathrm{R}}} \mathcal{U} \frac{\partial \mathcal{J}^{-1} \mathcal{U}}{\partial t} \mathrm{~d} \xi=-\int_{\xi_{\mathrm{L}}}^{\xi_{\mathrm{R}}} \mathcal{U} \frac{\partial \mathcal{U}}{\partial \xi} \mathrm{d} \xi
$$


We define the following norm, to be used in the sequel,

$$
\|\mathcal{U}\|^{2}=\int_{\xi_{\mathrm{L}}}^{\xi_{\mathrm{R}}} \mathcal{U}^{2} \mathcal{J}^{-1} \mathrm{~d} \xi .
$$

Using integration by parts on the right term of (3), bringing $\mathcal{U}$ within the temporal derivative and applying Leibniz's rule results in

$$
\frac{\mathrm{d}\|\mathcal{U}\|^{2}}{\mathrm{~d} t}=-\left.\mathcal{U}^{2}\right|_{\xi_{\mathrm{L}}} ^{\xi_{\mathrm{R}}}
$$

Applying the boundary condition, integrating in time, applying the initial condition and rearranging, we get that

$$
\|\mathcal{U}\|^{2} \leq\|\mathcal{F}\|^{2}+\int_{\tau=0}^{t} \mathcal{G}_{L}^{2} \mathrm{~d} \tau,
$$

which shows that the solution is bounded by the initial condition and the boundary data; therefore, the problem is stable [19].

A first-derivative SBP operator, applicable to general nodal distributions, is defined as [3]:

Definition 1. Summation-by-parts operator for the first derivative: A matrix operator, $\mathrm{D}_{\xi} \in \mathbb{R}^{N \times N}$, is an SBP operator, of global degree $p$, approximating the derivative $\frac{\partial}{\partial \xi}$, on the nodal distribution $\boldsymbol{\xi}$ in computational space, if

1. $\mathrm{D}_{\xi} \boldsymbol{\xi}^{k}=\mathrm{H}_{\xi}^{-1} \mathrm{Q}_{\xi} \boldsymbol{\xi}^{k}=\mathrm{H}_{\xi}^{-1}\left(\mathrm{~S}_{\xi}+\frac{1}{2} \mathrm{E}_{\xi}\right) \boldsymbol{\xi}^{k}=k \boldsymbol{\xi}^{k-1}, \quad k=0,1, \ldots, p ;$

2. $\mathrm{H}_{\xi}$, denoted the norm matrix, is symmetric positive definite;

3. $\mathrm{E}_{\xi}=\mathrm{E}_{\xi}^{\mathrm{T}}, \mathrm{S}_{\xi}=-\mathrm{S}_{\xi}^{\mathrm{T}}$, therefore, $\mathrm{Q}_{\xi}+\mathrm{Q}_{\xi}^{\mathrm{T}}=\mathrm{E}_{\xi}$; and

4. $\left(\boldsymbol{\xi}^{i}\right)^{\mathrm{T}} \mathrm{E}_{\xi} \boldsymbol{\xi}^{j}=\xi_{\mathrm{R}}^{i+j}-\xi_{\mathrm{L}}^{i+j}, \quad i, j=0,1, \ldots, r, r \geq p$.

This definition of $\mathrm{E}_{\xi}$ allows for nodal distributions that do not contain boundary nodes. For the purpose of imposing boundary conditions using SATs, it is convenient to further decompose $\mathrm{E}_{\xi}$ as [3]

$$
\mathrm{E}_{\xi}=\boldsymbol{t}_{\xi, R} \boldsymbol{t}_{\xi, R}^{\mathrm{T}}-\boldsymbol{t}_{\xi, L} \boldsymbol{t}_{\xi, L}^{\mathrm{T}}
$$

where

$$
\boldsymbol{t}_{\xi, R}^{\mathrm{T}} \boldsymbol{\xi}^{k}=\xi_{\mathrm{R}}^{k}, \quad \boldsymbol{t}_{\xi, L} \boldsymbol{\xi}^{k}=\xi_{\mathrm{L}}^{k}, \quad k=0,1, \ldots, r
$$


For operators constructed on nodal distributions that contain boundary nodes $\mathrm{E}_{\xi}$ can take a particularly simple form, given by

$$
\mathrm{E}_{\xi}=\operatorname{diag}(-1,0, \ldots, 0,1),
$$

where

$$
\boldsymbol{t}_{\xi, L}=[1,0, \ldots, 0]^{\mathrm{T}}, \quad \boldsymbol{t}_{\xi, R}=[0, \ldots, 0,1]^{\mathrm{T}},
$$

which is the form used in this paper.

The discretization of (1) is given by

$$
\frac{\mathrm{dJ}^{-1} \boldsymbol{u}_{h}}{\mathrm{~d} t}=-\mathrm{D}_{\xi} \boldsymbol{u}_{h}+\sigma \mathrm{H}_{\xi}^{-1}\left(\boldsymbol{t}_{\xi, L} \boldsymbol{t}_{\xi, L}^{\mathrm{T}} \boldsymbol{u}_{h}-\mathcal{G}_{L} \boldsymbol{t}_{\xi, L}\right)
$$

where $\mathrm{J}$ is a diagonal matrix with the Jacobian $\mathcal{J}$ evaluated at the mesh nodes along its diagonal. The last term is the SAT used to impose the boundary condition weakly $[6,7,8,9]$, and the scalar $\sigma$ is chosen such that the resulting discretization is stable and conservative. The norm matrix, $\mathrm{H}_{\xi}$, of an SBP operator induces an approximation to the $L_{2}$ inner product, such that $[3,20]$

$$
\boldsymbol{v}^{\mathrm{T}} \mathrm{H}_{\xi} \boldsymbol{u} \approx \int_{\xi_{\mathrm{L}}}^{\xi_{\mathrm{R}}} \mathcal{V U} \mathrm{d} \xi
$$

Thus, the discrete analogue of multiplying by the solution and integrating in computational space is multiplying $(7)$ by $\boldsymbol{u}_{h}^{\mathrm{T}} \mathrm{H}_{\xi}$, which results in

$$
\boldsymbol{u}_{h}^{\mathrm{T}} \mathrm{H}_{\xi} \frac{\mathrm{dJ}^{-1} \boldsymbol{u}_{h}}{\mathrm{~d} t}=-\boldsymbol{u}_{h}^{\mathrm{T}} \mathrm{Q}_{\xi} \boldsymbol{u}_{h}+\sigma \boldsymbol{u}_{h}^{\mathrm{T}}\left(\boldsymbol{t}_{\xi, L} \boldsymbol{t}_{\xi, L}^{\mathrm{T}} \boldsymbol{u}_{h}-\mathcal{G}_{L} \boldsymbol{t}_{\xi, L}\right) \text {. }
$$

Property 3 of an SBP operator, $\mathrm{Q}_{\xi}+\mathrm{Q}_{\xi}^{\mathrm{T}}=\mathrm{E}_{\xi}$, implies that $\boldsymbol{u}_{h}^{\mathrm{T}} \mathrm{Q}_{\xi} \boldsymbol{u}_{h}=$ $\frac{1}{2} \boldsymbol{u}_{h}^{\mathrm{T}} \mathrm{E}_{\xi} \boldsymbol{u}_{h}$. Furthermore, $\frac{1}{2} \frac{\mathrm{d} \boldsymbol{u}_{h}^{\mathrm{T}} \mathrm{H}_{\xi} \mathrm{J}^{-1} \boldsymbol{u}_{h}}{\mathrm{~d} t}=\boldsymbol{u}_{h}^{\mathrm{T}} \mathrm{H}_{\xi} \frac{\mathrm{dJ}^{-1} \boldsymbol{u}_{h}}{\mathrm{~d} \boldsymbol{t}}$, therefore, (8) becomes

$$
\frac{\mathrm{d}\left\|\boldsymbol{u}_{h}\right\|_{\mathrm{H}_{\xi} \mathrm{J}^{-1}}^{2}}{\mathrm{~d} t}=-\boldsymbol{u}_{h}^{\mathrm{T}}\left(\boldsymbol{t}_{\xi, R} \boldsymbol{t}_{\xi, R}^{\mathrm{T}}-\boldsymbol{t}_{\xi, L} \boldsymbol{t}_{\xi, L}^{\mathrm{T}}\right) \boldsymbol{u}_{h}+2 \sigma \boldsymbol{u}_{h}^{\mathrm{T}}\left(\boldsymbol{t}_{\xi, L} \boldsymbol{t}_{\xi, L}^{\mathrm{T}} \boldsymbol{u}_{h}-\mathcal{G}_{L} \boldsymbol{t}_{\xi, L}\right),
$$

where

$$
\left\|\boldsymbol{u}_{h}\right\|_{\mathrm{H}_{\xi} \mathrm{J}^{-1}}^{2}=\boldsymbol{u}_{h}^{\mathrm{T}} \mathrm{H}_{\xi} \mathrm{J}^{-1} \boldsymbol{u}_{h},
$$

and represents a norm if the norm matrix, $\mathrm{H}_{\xi}$, is diagonal [21]. Finally, completing the square, integrating in time and introducing the initial condition, with some rearrangement, (9) reduces to

$$
\left\|\boldsymbol{u}_{h}\right\|_{\mathrm{H}_{\xi} \mathrm{J}^{-1}}^{2} \leq\|\boldsymbol{f}\|_{\mathrm{H}_{\xi} \mathrm{J}^{-1}}^{2}-\gamma \Gamma^{2} \int_{\tau=0}^{t} \mathcal{G}_{L}^{2} \mathrm{~d} \tau
$$


where $\Gamma=\frac{-\sigma}{2 \sigma+1}$ and $\gamma=2 \sigma+1<0$ for stability. For conservation, $\sigma=-1$, and the final estimate is

$$
\left\|\boldsymbol{u}_{h}\right\|_{\mathrm{H}_{\xi} J^{-1}}^{2} \leq\|\boldsymbol{f}\|_{\mathrm{H}_{\xi} J^{-1}}^{2}+\int_{\tau=0}^{t} \mathcal{G}_{L}^{2} \mathrm{~d} \tau,
$$

which mimics the continuous estimate, (4), term by term.

We emphasize that the SBP property allows the discrete analysis to mimic the continuous analysis to the point before the boundary conditions are inserted. The SATs allow the introduction of the boundary conditions and allow one to mimic the continuous analysis for the remaining steps. It is, therefore, the combination of the properties of SBP operators and the weak imposition of the boundary conditions using SATs that allows the determination of the conditions under which the discretization is stable in a one-to-one fashion to the continuous analysis.

\section{Element-type implementation of SBP operators}

In this paper the focus is on operators that have a repeating interiorpoint operator, meaning that a sequential subset of nodes on the interior of the domain have the same difference operator. Such SBP operators can be implemented in three different ways.

For the purpose of discussion, consider the simplest classical SBP operator of global degree 1 with unit mesh spacing in computational space

$$
\begin{aligned}
& \mathrm{D}_{\xi}=\left[\begin{array}{ccccc}
-1 & 1 & & & \\
-\frac{1}{2} & 0 & \frac{1}{2} & & \\
& \ddots & \ddots & \ddots & \\
& & -\frac{1}{2} & 0 & \frac{1}{2} \\
& & & -1 & 1
\end{array}\right], \quad \mathrm{H}_{\xi}=\left[\begin{array}{ccccc}
\frac{1}{2} & & & & \\
& 1 & & & \\
& & \ddots & & \\
& & & 1 & \\
& & & \frac{1}{2}
\end{array}\right] \text {, } \\
& \mathrm{Q}_{\xi}=\left[\begin{array}{ccccc}
-\frac{1}{2} & \frac{1}{2} & & & \\
-\frac{1}{2} & 0 & \frac{1}{2} & & \\
& \ddots & \ddots & \ddots & \\
& & -\frac{1}{2} & 0 & \frac{1}{2} \\
& & & -\frac{1}{2} & \frac{1}{2}
\end{array}\right]
\end{aligned}
$$

In the traditional finite-difference approach, the operator (10) is applied on a set of $n$ nodes, the locations of which, in computational space, are 
given by $\xi_{i}=i, i \in[1, N]$. Mesh refinement is then accomplished by increasing the number of nodes. The operator is expanded by correspondingly increasing the number of rows that contain the interior-point operator $\left[\begin{array}{lllllll}\ldots & 0 & -\frac{1}{2} & 0 & \frac{1}{2} & 0 & \ldots\end{array}\right]$.

Alternatively, the domain can be divided into a number of elements, and the SBP operator in each element is of fixed size $[3,4,10,22]$. Mesh refinement is accomplished by increasing the number of elements. We have two choices to construct a global derivative operator, the discontinuous and continuous-element approaches. In the discontinuous approach, the global first derivative operator is assembled by inserting the local derivative operators. For example, having 3 nodes in each element, the global derivative operator for $K$ elements is given as

$$
\mathrm{D}_{\xi, g}=\left[\begin{array}{cccc}
\mathrm{D}_{\xi}^{(1)} & & & \\
& \mathrm{D}_{\xi}^{(2)} & & \\
& & \ddots & \\
& & & \mathrm{D}_{\xi}^{(K)}
\end{array}\right], \quad \mathrm{D}_{\xi}^{(i)}=\left[\begin{array}{ccc}
-1 & 1 & \\
-\frac{1}{2} & 0 & \frac{1}{2} \\
& -1 & 1
\end{array}\right],
$$

and the superscript refers to the element number. In this approach, the global derivative operator does not have coupling between elements and a mechanism to introduce coupling is necessary. If the nodal distribution in each element contains boundary nodes, then one can use the continuous element approach [10]; however, in this paper, we will only consider the traditional finite-difference and discontinuous approaches.

\section{Construction of corner-corrected operators}

As discussed, one of the drawbacks of diagonal-norm classical FD-SBP operators is that while the interior operator is of degree $2 p$, a number of point operators, for nodes at and near the boundary, are of degree $p$; therefore the operator is of global degree $p$, rendering the method order $p+1$ for hyperbolic problems. Here we propose a modification of the form of classical FD-SBP operators that allows us to produce operators that are of global degree $2 p$. This requires two modifications: first, as discussed below, it is necessary to modify the form of the operator, and second, mesh refinement must be carried out using the element approach.

To allow for compact presentation, the modifications and the steps used to develop this new class of operators are exemplified using the classical FDSBP operators with a degree 2 interior-point operator. The norm matrix of 
the classical operator, $\mathrm{H}_{\xi}$, and the corner-corrected operator, $\tilde{\mathrm{H}}_{\xi}$, for six-node operators are generically given by

$$
\begin{aligned}
& \mathrm{H}_{\xi}=\operatorname{diag}\left(h_{11}, h_{22}, 1,1, h_{22}, h_{11}\right), \\
& \tilde{\mathrm{H}}_{\xi}=\operatorname{diag}\left(\tilde{h}_{11}, \tilde{h}_{22}, 1,1, \tilde{h}_{22}, \tilde{h}_{11}\right),
\end{aligned}
$$

where for the classical operator $h_{11}=1 / 2$ and $h_{22}=1$. The generic representation of both $S_{\xi}$ and $\tilde{S}_{\xi}$ is given as

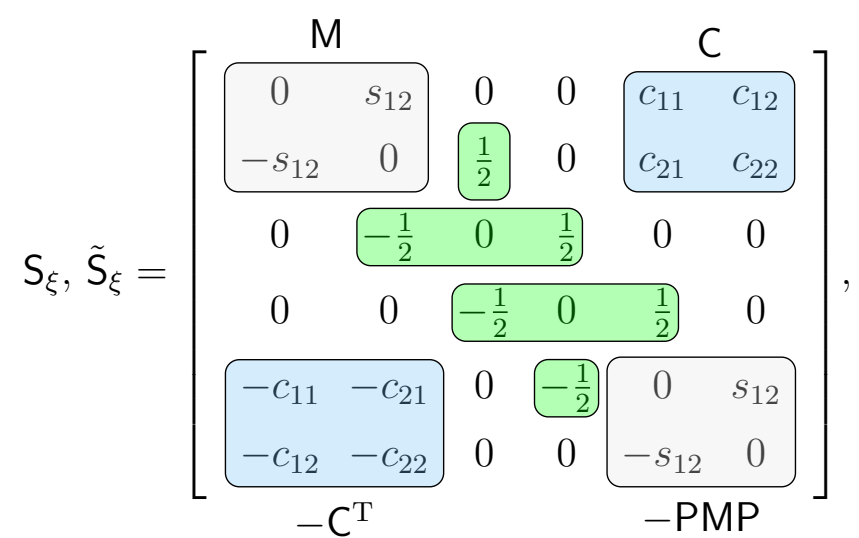

where $P$ is a square matrix with ones along the anti-diagonal; this means that -PMP is the negative of the permutation of the rows and columns of $\mathrm{M}$. The contributions from the interior-point operator are highlighted in green. In order to satisfy the SBP property $\mathrm{Q}_{\xi}+\mathrm{Q}_{\xi}^{\mathrm{T}}=\mathrm{E}_{\xi}, \mathrm{M}$ must be skew symmetric, as depicted in (12). The matrix $C$ is added such that the degree of the operator for boundary nodes can be increased. In order to maintain the SBP property, the correction for the right boundary nodes must be the negative transpose of the correction for the left boundary nodes. The matrix $M$ for the corner-corrected operator is not the same as for the classical operator. Although corner-corrected operators of arbitrary size can be constructed, the matrices $\mathrm{M}$ and $\mathrm{C}$ depend on $N$. Hence, mesh refinement must be carried out using the element approach for the modified derivative operator $\tilde{D}_{\xi}$ to remain an approximation to the derivative. The classical operator is recovered from (12) by setting $\mathrm{C}=0$ and $s_{12}=1 / 2$.

We seek to construct general corner-corrected operators of arbitrary degree and size. Specifically, we seek general solutions that lead to unique 
expressions for the coefficients of the norm matrix; this is advantageous as it 155 is then possible to determine the values of $N$ that lead to corner-corrected operators having a positive-definite norm matrix, necessary for stability. While operators with boundary point-operators of degree $p$ through $2 p$ can be constructed, we limit the exposition to operators that are uniformly of degree $2 p$. To demonstrate the procedure for deriving corner-corrected operators, we do so using operators with an interior-point operator of degree two, i.e., at interior nodes, the operator has a stencil centered on the diagonal $\left[-\frac{1}{2}, 0, \frac{1}{2}\right]$. In general, we use $4 p$ non-unity weights for the norm matrix for operators of uniform $2 p$ degree. In the present example, the nodal distribution is uniform and contains nodes on the boundary; therefore, $\mathrm{E}_{\xi}=\operatorname{diag}(-1,0, \ldots, 0,1)$. Moreover, $\tilde{H}_{\xi}$ is given in (11), and the form of $\tilde{S}_{\xi}$ is given in (12), where for operators with more than six nodes, additional interior-point operators are added, while the $\mathrm{C}$ matrices remain in the corners.

The corner-corrected operator must satisfy the degree conditions (1) in Definition 1. To develop general solutions, the degree conditions have to be set up such that the number of nodes enters the derivation. Given that the interior stencil is constructed to be of degree $2 p$, only the boundary operators need to be determined. Taking the number of nodes in the element as $N$, the degree conditions that must be solved can be developed by constructing $\boldsymbol{\xi}$ as

$$
\boldsymbol{\xi}=[1,2, \ldots, 3 p, N-3 p+1, \ldots, N-1, N]^{\mathrm{T}} .
$$

We use $3 p$ nodes at either boundary so that the coefficients of each stencil that are not the corner corrections only interact with their respective boundary nodes. The first and last $2 p$ degree conditions must be solved for. For the current example, $\boldsymbol{\xi}=[1,2,3, N-2, N-1, N]^{\mathrm{T}}$. This leads to the following four degree conditions for $k=0$ :

$$
\begin{aligned}
& \text { Node } 1: \quad-\frac{1}{2}+s_{12}+c_{11}+c_{12}=0 \\
& \text { Node } 2: \quad \frac{1}{2}+-s_{12}+c_{21}+c_{22}=0 \\
& \text { Node } N-1: \quad-\frac{1}{2}-c_{11}-c_{21}+s_{12}=0 \\
& \text { Node } N: \quad \frac{1}{2}-c_{12}-c_{22}-s_{12}=0
\end{aligned}
$$


For $k=1$, the degree conditions are

$$
\text { Node 1: } \quad-\frac{1}{2}+2 s_{12}+c_{11}(N-1)+c_{12} N-\tilde{h}_{11}=0
$$

Node 2: $\quad-s_{12}+\frac{3}{2}+c_{21}(N-1)+c_{22} N-\tilde{h}_{22}=0$

Node $N-1: \quad-c_{11}-2 c_{21}+1-\frac{1}{2} N+s_{12} N-\tilde{h}_{22}=0$

Node $N: \quad-c_{12}-2 c_{22}-s_{12}(N-1)+\frac{1}{2} N-\tilde{h}_{11}=0$

and for $k=2$,

Node 1: $\quad-\frac{1}{2}+4 s_{12}+c_{11}(N-1)^{2}+c_{12} N^{2}-2 \tilde{h}_{11}=0$

Node $2: \quad-s_{12}+\frac{9}{2}+c_{21}(N-1)^{2}+c_{22} N^{2}-4 \tilde{h}_{22}=0$

Node $N-1: \quad-c_{11}-4 c_{21}-\frac{1}{2}(N-2)^{2}+s_{12} N^{2}-2 \tilde{h}_{22}(N-1)=0$

Node $N: \quad-c_{12}-4 c_{22}-s_{12}(N-1)^{2}+\frac{1}{2} N^{2}-2 \tilde{h}_{11} N=0$

In the above equations, $N$ is treated as a constant, and the equations are therefore linear, the solution of which is

$$
\begin{aligned}
& c_{11}=-\frac{1}{12} \frac{N-4}{N-2}, \quad c_{12}=\frac{1}{12} \frac{N^{2}-8 N+13}{N^{2}-4 N+4}, \quad c_{21}=\frac{1}{12} \frac{N^{2}-4 N+3}{N^{2}-4 N+4}, \\
& c_{22}=-\frac{1}{12} \frac{N-4}{N-2}, \quad \tilde{h}_{11}=\frac{1}{12} \frac{5 N-11}{N-2}, \quad \tilde{h}_{22}=\frac{1}{12} \frac{13 N-25}{N-2}, \\
& s_{12}=\frac{1}{12} \frac{6 N^{2}-22 N+19}{N^{2}-4 N+4} .
\end{aligned}
$$

For example, on six nodes we get

$$
\tilde{\mathrm{H}}_{\xi}=\operatorname{diag}\left(\frac{19}{48}, \frac{53}{48}, 1,1, \frac{53}{48}, \frac{19}{48}\right),
$$




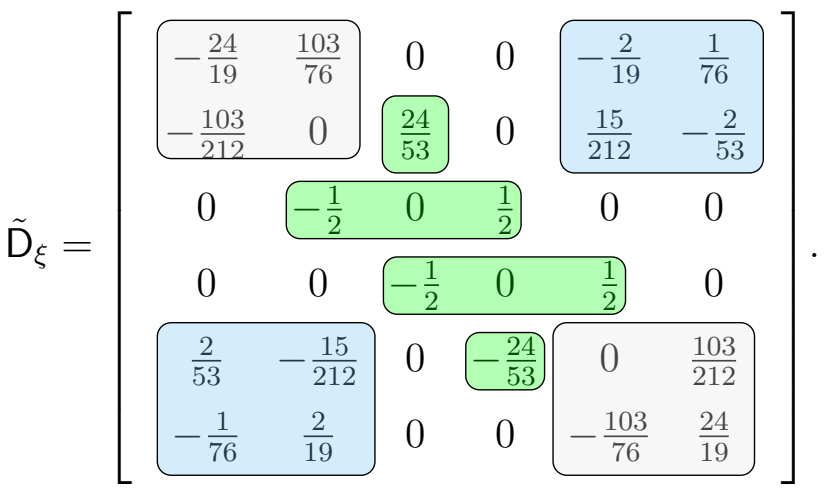

In this case, the minimum number of required nodes to construct the cornercorrected operator is five, and in general, the minimum number of nodes for uniform nodal distributions is $5 p$. In the present example, we have constructed the operator to have a global degree that matches the degree of the interior-point operator, which is $2 p$. It is possible to construct cornercorrected operators with global degree less than $2 p$ and for such operators the minimum number of nodes can be decreased. The general solution leads to a positive definite norm matrix for $N$ greater than or equal to this minimum. For this example, the solution to the degree equations is unique. In general, however, free parameters remain and must be determined somehow. This naturally leads to the concept of optimization. The norm matrix of SBP operators is used to approximate the $L_{2}$ norm of the error and functionals of the solution. Therefore, the discrete inner product of the error of the first monomial not satisfied by the degree conditions is used as an objective function:

$$
J_{\boldsymbol{e}}=\left\|\boldsymbol{e}_{\tilde{p}}\right\|_{\tilde{\mathrm{H}}_{\xi}}^{2}=\boldsymbol{e}_{2 p+1}^{\mathrm{T}} \tilde{\mathrm{H}}_{\xi} \boldsymbol{e}_{2 p+1},
$$

where the error vector is given by

$$
\boldsymbol{e}_{2 p+1}=\tilde{\mathrm{D}}_{\xi} \boldsymbol{\xi}^{2 p+1}-(2 p+1) \boldsymbol{\xi}^{2 p} .
$$

Minimizing $J_{e}$ typically does not determine all of the free parameters. Furthermore, without additional constraints, some of the operators that result have very large coefficients and are therefore susceptible to round-off error. For example, the operator of global degree 6 on a uniform nodal distribution without any optimization and setting all free parameters to 0 has values of the largest absolute value of the difference matrix increasing from 3465 to 54322 , for $N=12$ to $N=50$. Therefore, in addition to (13), we employ a

second objective function, $J_{\tilde{\mathrm{D}}_{\xi}}$, which is the sum of the squares of the entries 
in $\tilde{\mathrm{D}}_{\xi}$, and in this way, the size of the coefficients is reduced. Using $J_{\tilde{\mathrm{D}}_{\xi}}$ has a secondary benefit, in that for all operators investigated in this paper, the remaining free parameters are specified and no further optimization is necessary. Free parameters that do not affect $J_{\boldsymbol{e}}$ are used to optimize $J_{\tilde{\mathrm{D}}_{\xi}}$, where Maple's ${ }^{\circledR}$ minimize function is used to analytically perform the optimization.

Now we summarize the required steps to derive corner-corrected operators:

- Solve the degree conditions for the first and last $2 p$ nodes for operators of degree $2 p$.

- Check that $\tilde{\mathrm{H}}_{\xi}$ is uniquely defined by $N$ and for what values of $N$ it is positive definite.

- If any free parameters remain, for each $N$ of interest, first optimize using $J_{e}$; if free parameters remain, optimize using $J_{\tilde{\mathrm{D}}_{\xi}}$.

Note that for all operators considered in this paper we achieved a unique $\tilde{\mathrm{H}}_{\xi}$. If free parameters remain in $\tilde{\mathrm{H}}_{\xi}$ one could optimize in some way with the constraint that $\tilde{\mathrm{H}}_{\xi}$ remain positive definite. With the exception of the cornercorrected operator of global degree eight, all remaining corner-corrected operators on uniform nodal distributions studied, i.e. global degree $2-7$, have positive definite $\mathrm{H}_{\xi}$ from the minimum required number of nodes necessary to construct the operators to $N=50$. We did find that the eight-order operator can result in positive norm matrices for $N \geq 57$.

\subsection{Hybrid-Gauss-trapezoidal and Lobatto operators}

In addition to constructing corner-corrected versions of SBP operators on uniform nodal distributions (UND), in computational space, we consider families of corner-corrected operators built on the hybrid-Gauss-trapezoidal (HGT) and hybrid-Gauss-trapezoidal-Lobatto (HGTL) quadrature nodes developed by Alpert [23]. This family of nodal distributions has a number of nodes near the boundaries that are unequally spaced with equally spaced interior nodes. The HGT family does not include nodes at the boundaries while the HGTL family does. These nodal distributions are similar in spirit to those proposed by Mattsson and Carpenter [24] and have been shown to lead to SBP operators with preferential error characteristics as compared to UND operators $[12,25]$ as a result of the smaller truncation error from the lower degree boundary point operators. 
The nodal locations and quadrature weights are derived from the solution to

$$
\sum_{i=1}^{j} \tilde{w}_{i} \tilde{x}_{i}^{r}=\frac{B_{r+1}(a)}{r+1}, \quad r=0,1, \ldots, 2 j-2,
$$

where $B_{i}(\xi)$ is the $i^{\text {th }}$ Bernoulli polynomial, $B_{0}(\xi)=1$. In order to include nodes at the boundaries, we supplement equations (15) with the condition $\tilde{x}_{0}=0$. The parameters $a$ and $j$ are chosen so that a particular degree is attained. If they are chosen such that $a=j$, which is the approach taken here, then it is possible to show that the resultant quadrature rules are positive definite up to degree 20 [23]. To construct a nodal distribution on $\xi \in[0,1]$, the following relations are used:

$$
\begin{aligned}
\xi_{i} & =h \tilde{x}_{i}, \quad \xi_{N-(i-1)}=1-h \tilde{x}_{i}, \quad i=1,2, \ldots, j \\
\xi_{i+j+1} & =h(a+i), \quad i=0,1, \ldots n-1,
\end{aligned}
$$

where $h=\frac{1}{n+2 j-1}, n$ is the number of uniformly distributed nodes, and the total number of nodes is given as $N=n+2 j$.

Instead of using the quadrature rules given by Alpert [23], we use his nodal distributions and construct $S_{\xi}$ and $\tilde{S}_{\xi}$ of form (12) on these nodal distributions. As a result of the unequal nodal distribution at the first few nodes, the interior-point operator can only be used starting at the $2 p^{\text {th }}$ node. This means that, in contrast to the uniform nodal distribution case, for corner-corrected operators of global degree less than $2 p$ the minimum number of nodes remains $5 p$. For example, the family of HGTL operators of degree four with $p=2$ on ten nodes, where the computational domain is $\xi \in[-1,1]$, have a nodal distribution

$$
\boldsymbol{\xi}=\left[-1,-\frac{31}{39},-\frac{5}{9},-\frac{1}{3},-\frac{1}{9}, \frac{1}{9}, \frac{1}{3}, \frac{5}{9}, \frac{31}{39}, 1\right]^{\mathrm{T}} .
$$

The interior point operator requires that the values of the function lie on equally distributed nodes, and examining the nodal distribution, it can be seen that this only occurs starting at the fifth node. For this nodal distribution, to two significant digits, the various matrices of the corner-corrected operator are

$$
\begin{aligned}
& \tilde{\mathrm{H}}_{\xi}=\operatorname{diag}(0.065,0.28,0.19,0.23,0.22,0.22,0.23,0.19,0.28,0.065), \\
& \boldsymbol{t}_{\xi, L}=[1,0 \ldots, 0]^{\mathrm{T}}, \quad \boldsymbol{t}_{\xi, R}=[0, \ldots, 0,1]
\end{aligned}
$$




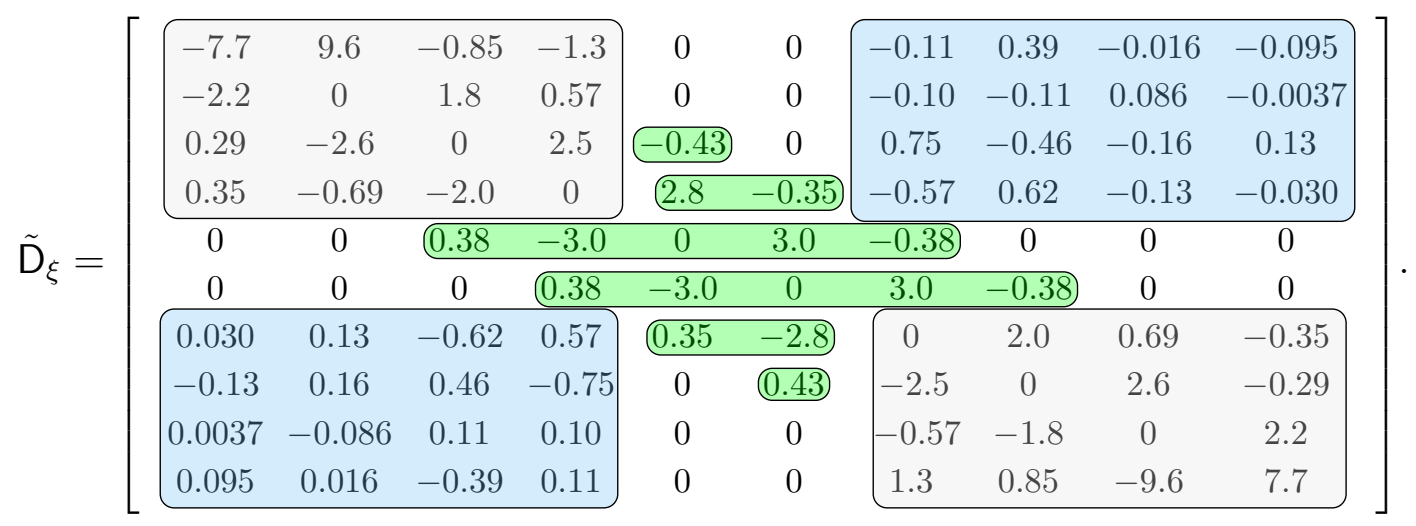

For the HGT operators we have a further issue in that it is necessary to construct $\boldsymbol{t}_{\xi, L}$ and $\boldsymbol{t}_{\xi, R}$ for the decomposition of $\mathrm{E}_{\xi}$ given in (5). Since the operators are of degree $2 p$ at all nodes, and $\boldsymbol{t}_{\xi, L}$ and $\boldsymbol{t}_{\xi, R}$ must satisfy $2 p+1$ conditions given in (6), the first and last $2 p+1$ rows of $\tilde{\mathbf{D}}_{\xi}$ are not the interior-point operator and therefore the minimum number of nodes for these operators is $5 p+2$; however, we have found that $\tilde{\mathrm{H}}_{\xi}$ only requires $4 p$ non-unity weights. For example, for operators of degree four $\boldsymbol{t}_{\xi, L}$ and $\boldsymbol{t}_{\xi, R}$ must satisfy five equations and therefore require coefficients for at least the first and last five nodes, respectively. For $p=2$ the minimum is twelve nodes, and the nodal distribution, to two significant digits, is

$$
\begin{array}{r}
\boldsymbol{\xi}=[-0.96,-0.82,-0.64,-0.45-0.27,-0.091 \\
0.091,0.27,0.45,0.64,0.82,0.96]
\end{array}
$$

where the computational domain is $\xi \in[-1,1]$. The norm matrix, to six significant digits, is given as

$$
\begin{aligned}
& \tilde{\mathrm{H}}_{\xi}=\operatorname{diag}(0.100751,0.171955,0.181853,0.181804,0.181818, \\
& \quad 0.181818,0.181818,0.181818,181804,0.181853,0.171955,0.100751) .
\end{aligned}
$$

To two significant digits, $\boldsymbol{t}_{\xi, L}, \boldsymbol{t}_{\xi, R}$, and $\tilde{\mathrm{D}}_{\xi}$ are

$$
\begin{aligned}
& \boldsymbol{t}_{\xi, L}=\left[\begin{array}{llllllllllll}
1.6 & -1.2 & 0.78 & -0.33 & 0.061 & 0.0 & 0.0 & 0.0 & 0.0 & 0.0 & 0.0 & 0.0
\end{array}\right],
\end{aligned}
$$

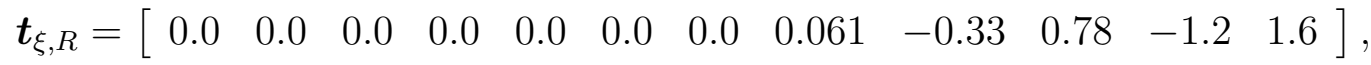




\begin{tabular}{l|c|c}
$\begin{array}{c}\text { degree of } \\
\text { interior-point } \\
\text { operator }\end{array}$ & global degree & $\begin{array}{c}\text { Minimum } \\
\text { element size } \\
\text { UND }\end{array}$ \\
\hline 2 & 1 & 4 \\
4 & 2 & 9 \\
6 & 3 & 13 \\
8 & 4 & 17 \\
10 & 5 & $23^{*}$ \\
12 & 6 & $29^{*}$ \\
14 & 7 & $39^{*}$ \\
16 & 8 & $47^{*}$
\end{tabular}

Table 1: Minimum element size for UND SBP operators (entries with * are taken from Ref. [13]).

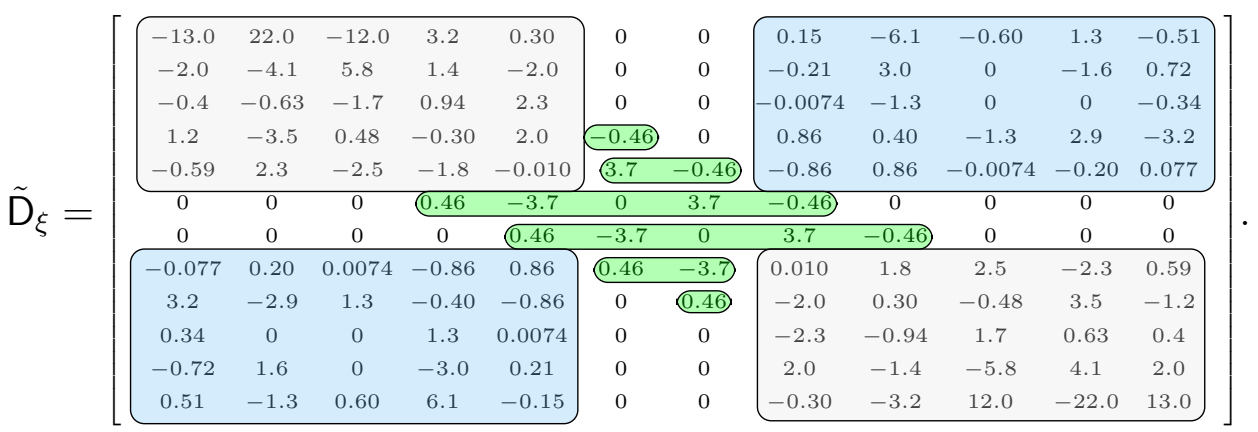

For both the HGT and HGTL operators, we found that the norm matrix was positive definite from the minimum number of nodes up to $N=50$ for operators of global degree $2,4,6$, and 8 .

We summarize the minimum number of nodes per element for classical SBP operators and corner-corrected operators in Tables 1 and 2; we can see that for the same global degree, the corner-corrected operators have a significantly smaller minimum number of nodes.

We provide Matlab $^{\circledR}$ for all the operators considered in this paper; see Appendix Appendix B.

\section{Results}

To compare the properties of the proposed first-derivative SBP operators we consider the two-dimensional linear convection equation on a unit square 


\begin{tabular}{l|c|c|c}
$\begin{array}{c}\text { degree of } \\
\text { interior-point } \\
\text { operator }\end{array}$ & global degree & $\begin{array}{c}\text { Minimum } \\
\text { element size } \\
\text { UND/HGTL }\end{array}$ & $\begin{array}{c}\text { Minimum } \\
\text { element size } \\
\text { HGT }\end{array}$ \\
\hline 2 & 2 & $5^{*}$ & - \\
4 & 4 & 10 & 12 \\
6 & 6 & 15 & 17 \\
8 & 8 & $20^{* *}$ & 22
\end{tabular}

Table 2: Minimum element size for corner-corrected operators; ${ }^{*}$ operator does not exist for HGTL;** the minimum size does not exist for the UND operators.

with unit velocity components:

$$
\frac{\partial \mathcal{U}}{\partial t}+\frac{\partial \mathcal{U}}{\partial x}+\frac{\partial \mathcal{U}}{\partial y}=0, \quad \forall(x, y) \in[0,1] \times[0,1], \quad t \geq 0 .
$$

The initial condition is a Gaussian:

$\mathcal{U}(x, y, 0)=\exp \left[-150\left(x-\frac{1}{2}\right)^{2}-150\left(y-\frac{1}{2}\right)^{2}\right], \quad \forall(x, y) \in[0,1] \times[0,1]$

with a value of less than $10^{-16}$ at the boundaries, which are periodic:

$$
\begin{aligned}
& \mathcal{U}(x, 0, t)=\mathcal{U}(x, 1, t) \\
& \mathcal{U}(0, y, t)=\mathcal{U}(1, y, t), \quad \forall(x, y) \in[0,1] \times[0,1], \quad t \geq 0
\end{aligned}
$$

The domain is partitioned into $K$ nonoverlapping elements. On each element, the PDE (16) is mapped from physical coordinates $(x, y)$ to rectilinear computational coordinates $(\xi, \eta)$, yielding the divergence form of the transformed PDE:

$$
\frac{\partial\left(\mathcal{J}^{-1} \mathcal{U}\right)}{\partial t}+\frac{\partial\left(\lambda_{\xi} \mathcal{U}\right)}{\partial \xi}+\frac{\partial\left(\lambda_{\eta} \mathcal{U}\right)}{\partial \eta}=0
$$

where

$$
\lambda_{\xi}=\frac{\partial y}{\partial \eta}-\frac{\partial x}{\partial \eta}, \quad \lambda_{\eta}=-\frac{\partial y}{\partial \xi}+\frac{\partial x}{\partial \xi}, \quad \mathcal{J}^{-1}=\frac{\partial x}{\partial \xi} \frac{\partial y}{\partial \eta}-\frac{\partial x}{\partial \eta} \frac{\partial y}{\partial \xi} .
$$

This can also be recast in skew-symmetric form

$$
\frac{\partial\left(\mathcal{J}^{-1} \mathcal{U}\right)}{\partial t}+\frac{1}{2} \frac{\partial\left(\lambda_{\xi} \mathcal{U}\right)}{\partial \xi}+\frac{1}{2} \frac{\partial\left(\lambda_{\eta} \mathcal{U}\right)}{\partial \eta}+\frac{\lambda_{\xi}}{2} \frac{\partial \mathcal{U}}{\partial \xi}+\frac{\lambda_{\eta}}{2} \frac{\partial \mathcal{U}}{\partial \eta}=0
$$


We are interested in the skew-symmetric form as it is a particular instance of a splitting of the PDE. Such forms are important in nonlinear stability, see Refs. [26, 27, 28], and they have been shown to improve robustness [29].

\subsection{Discretization}

The decomposition of the domain into elements is Cartesian, with each element discretized with the nodal distribution associated with the operator applied. In this case, the divergence (18) and skew-symmetric (20) forms are equivalent as the metrics are constant throughout the domain. The divergence form is less computationally intensive and is therefore applied for simulations on Cartesian meshes. In Appendix A results on a curved mesh are presented, in which case the skew-symmetric form (20) is used.

The SBP-SAT semi-discretization of (20) is now presented. In this article, periodicity is enforced with interface SATs between periodic faces. Therefore, to simplify the presentation a two block discretization is discussed with a single shared interface of constant $\xi$. The subscripts $L$ and $R$ will be used to denote the operators in the left and right elements, respectively. It has been assumed that in the $\eta$ direction the two elements are conforming and have the same nodal distribution. The discretization in the left element is given as

$$
\begin{aligned}
\frac{\mathrm{dJ}_{L}^{-1} \boldsymbol{u}_{L}}{\mathrm{~d} t}+ & \frac{1}{2}\left(\mathrm{D}_{\xi_{L}} \otimes \mathrm{I}_{\eta}\right) \Lambda_{\xi_{L}} \boldsymbol{u}_{L}+\frac{1}{2}\left(\mathrm{I}_{L, \xi} \otimes \mathrm{D}_{\eta}\right) \Lambda_{\eta_{L}} \boldsymbol{u}_{L} \\
+ & \frac{1}{2} \Lambda_{\xi_{L}}\left(\mathrm{D}_{\xi_{L}} \otimes \mathrm{I}_{\eta}\right) \boldsymbol{u}_{L}+\frac{1}{2} \Lambda_{\eta_{L}}\left(\mathrm{I}_{L, \xi} \otimes \mathrm{D}_{\eta}\right) \boldsymbol{u}_{L}= \\
& \frac{1}{4}\left(\mathrm{H}_{\xi_{L}}^{-1} \boldsymbol{t}_{L, \xi_{1}} \otimes \Sigma_{\mathrm{L}}\right)\left[\left(\boldsymbol{t}_{L, \xi_{1}}^{\mathrm{T}} \otimes \mathrm{I}_{\eta}\right) \Lambda_{\xi_{L}} \boldsymbol{u}_{L}-\left(\boldsymbol{t}_{R, \xi_{0}}^{\mathrm{T}} \otimes \mathrm{I}_{\eta}\right) \Lambda_{\xi_{R}} \boldsymbol{u}_{R}\right] \\
& +\frac{1}{4} \Lambda_{\xi_{L}}\left(\mathrm{H}_{\xi_{L}}^{-1} \boldsymbol{t}_{L, \xi_{1}} \otimes \Sigma_{\mathrm{L}}\right)\left[\left(\boldsymbol{t}_{L, \xi_{1}}^{\mathrm{T}} \otimes \mathrm{I}_{\eta}\right) \boldsymbol{u}_{L}-\left(\boldsymbol{t}_{R, \xi_{0}}^{\mathrm{T}} \otimes \mathrm{I}_{\eta}\right) \boldsymbol{u}_{R}\right]
\end{aligned}
$$

where $\Sigma_{\mathrm{L}}$ is a diagonal matrix of SAT coefficients discussed below. The discrete metric components are constructed as follows

$$
\begin{aligned}
& \Lambda_{\xi_{L}}=\operatorname{diag}\left[\left(\mathrm{I}_{L, \xi} \otimes \mathrm{D}_{\eta}\right) \boldsymbol{y}_{L}-\left(\mathrm{I}_{L, \xi} \otimes \mathrm{D}_{\eta}\right) \boldsymbol{x}_{L}\right] \\
& \Lambda_{\eta_{L}}=\operatorname{diag}\left[-\left(\mathrm{D}_{\xi_{L}} \otimes \mathrm{I}_{\eta}\right) \boldsymbol{y}_{L}+\left(\mathrm{D}_{\xi_{L}} \otimes \mathrm{I}_{\eta}\right) \boldsymbol{x}_{L}\right] \\
& \mathrm{J}_{L}^{-1}=\operatorname{diag}\left[\left(\mathrm{D}_{\xi_{L}} \otimes \mathrm{I}_{\eta}\right) \boldsymbol{x}_{L} \odot\left(\mathrm{I}_{L, \xi} \otimes \mathrm{D}_{\eta}\right) \boldsymbol{y}_{L}-\left(\mathrm{I}_{L, \xi} \otimes \mathrm{D}_{\eta}\right) \boldsymbol{x}_{L} \odot\left(\mathrm{D}_{\xi_{L}} \otimes \mathrm{I}_{\eta}\right) \boldsymbol{y}_{L}\right]
\end{aligned}
$$


where $\odot$ denotes the Hadamard product. Likewise, the discretization in the right element is given as

$$
\begin{aligned}
\frac{\mathrm{d} J_{R}^{-1} \boldsymbol{u}_{R}}{\mathrm{~d} t}+ & \frac{1}{2}\left(\mathrm{D}_{\xi_{R}} \otimes \mathrm{I}_{\eta}\right) \Lambda_{\xi_{R}} \boldsymbol{u}_{R}+\frac{1}{2}\left(\mathrm{I}_{R, \xi} \otimes \mathrm{D}_{\eta}\right) \Lambda_{\eta_{R}} \boldsymbol{u}_{R} \\
+ & \frac{1}{2} \Lambda_{\xi_{R}}\left(\mathrm{D}_{\xi_{R}} \otimes \mathrm{I}_{\eta}\right) \boldsymbol{u}_{R}+\frac{1}{2} \Lambda_{\eta_{R}}\left(\mathrm{I}_{R, \xi} \otimes \mathrm{D}_{\eta}\right) \boldsymbol{u}_{R}= \\
& \frac{1}{4}\left(\mathrm{H}_{\xi_{R}}^{-1} \boldsymbol{t}_{R, \xi_{0}} \otimes \Sigma_{\mathrm{R}}\right)\left[\left(\boldsymbol{t}_{R, \xi_{0}}^{\mathrm{T}} \otimes \mathrm{I}_{\eta}\right) \Lambda_{\xi_{R}} \boldsymbol{u}_{R}-\left(\boldsymbol{t}_{L, \xi_{1}}^{\mathrm{T}} \otimes \mathrm{I}_{\eta}\right) \Lambda_{\xi_{L}} \boldsymbol{u}_{L}\right] \\
& +\frac{1}{4} \Lambda_{\xi_{R}}\left(\mathrm{H}_{\xi_{R}}^{-1} \boldsymbol{t}_{R, \xi_{0}} \otimes \Sigma_{\mathrm{R}}\right)\left[\left(\boldsymbol{t}_{R, \xi_{0}}^{\mathrm{T}} \otimes \mathrm{I}_{\eta}\right) \boldsymbol{u}_{R}-\left(\boldsymbol{t}_{L, \xi_{1}}^{\mathrm{T}} \otimes \mathrm{I}_{\eta}\right) \boldsymbol{u}_{L}\right] .
\end{aligned}
$$

There are two common choices for the coefficients in the SAT matrices $\Sigma_{\mathrm{L}}$ and $\Sigma_{\mathrm{R}}$. The first can be thought of as using an upwind flux function, and has the form

$$
\Sigma_{\mathrm{L}}=\frac{-\tilde{\Lambda}_{L, \xi}+\left|\tilde{\Lambda}_{L, \xi}\right|}{\left|\tilde{\Lambda}_{L, \xi}\right|}, \quad \Sigma_{\mathrm{R}}=\frac{-\tilde{\Lambda}_{R, \xi}-\left|\tilde{\Lambda}_{R, \xi}\right|}{\left|\tilde{\Lambda}_{R, \xi}\right|},
$$

where $\tilde{\Lambda}_{L, \xi}=\left(\boldsymbol{t}_{L, \xi_{1}}^{\mathrm{T}} \otimes \mathrm{I}_{\eta}\right) \Lambda_{\xi_{L}}\left(\mathbf{1}_{L, \xi} \otimes \mathrm{I}_{\eta}\right), \tilde{\Lambda}_{R, \xi}=\left(\boldsymbol{t}_{R, \xi_{1}}^{\mathrm{T}} \otimes \mathrm{I}_{\eta}\right) \Lambda_{\xi_{R}}\left(\mathbf{1}_{R, \xi} \otimes \mathrm{I}_{\eta}\right)$, and 1 denotes a column vector of ones. The second is typically referred to as a symmetric SAT, and has the form

$$
\Sigma_{\mathrm{L}}=\mathrm{I}_{\eta}, \quad \Sigma_{\mathrm{R}}=-\mathrm{I}_{\eta} .
$$

Symmetric SATs are less dissipative; however, they sometimes lead to underconvergence when using the element approach $[4,30]$. Therefore, the focus in this article will be on results obtained with upwind SATs. The extension of the discretization method presented here to systems of PDEs can be found elsewhere, for example Refs. [31, 32, 33].

The following conventions are used in reference to SBP operators:

- A subscript "cc" is appended to refer to corner-corrected operators; for example, $\mathrm{UND}_{\mathrm{cc}}$ refers to the corner-corrected operators on a uniform nodal distribution.

- A subscript "trad" is used to denote use of the traditional finite-difference approach to mesh refinement. 
- The degree of the interior-point operator and global degree of an operator is given by appending an ordered pair after the acronym; for example, $\operatorname{HGTL}(\tilde{p}, p)$ refers to the hybrid-Gauss-trapezoidal-Lobatto SBP operator with an interior-point operator of degree $\tilde{p}$ and global degree of $p$. If the operator is of uniform pointwise degree, only a single value is given; for example, $\mathrm{HGTL}_{\mathrm{cc}}(6)$ refers to a corner corrected HGTL operator of degree 6.

- A numerical subscript is used to denote the number of degrees of freedom $N$ in the operator used; for example, $\mathrm{UND}_{5}(2,1)$ refers to a classical FD-SBP operator of global degree 1 with 5 degrees of freedom.

\subsection{Spectral Radii}

255 This section investigates the spectral radii of the spatial discretization matrix of the proposed corner-corrected SBP operators in relation to classical SBP operators. The spectral radius of the spatial discretization matrix dictates the maximum stable time step for explicit time-marching methods. This can have a significant impact on the relative efficiency obtained, especially for time-stability limited simulations. This comparison does not account for the relative accuracy of the methods, which will be addressed in a subsequent section.

When scaled by the average mesh spacing, the spectral radius asymptotes to a constant value. This holds for both traditional and discontinuouselement approaches to grid refinement. Table 3 shows the scaled spectral radius computed on Cartesian meshes, and organized by the degree of the interior-point operator $\tilde{p}$. For curved meshes, it was found that the spectral radius was simply a constant multiple of the spectral radius computed on the Cartesian mesh. Using the discontinuous-element refinement strategy, this multiple was the same for all operators considered. Therefore, only the spectral radii computed on Cartesian meshes are presented.

In most cases, the corner-corrected UND and HGTL operators have a similar scaled spectral radius to their classical counterparts. This is significant in two ways. First, it demonstrates that the corner corrections do not have a significant impact on the spectral radius of the operators. Secondly, with a similar spectral radius, the corner-corrected operators obtain twice the global degree $p$. If instead we compare the operators for the same global degree $p$, the corner-corrected operators have smaller spectral radii than the classical SBP operators. 


\begin{tabular}{l|l} 
Operator & $\rho_{\text {asym }}$ \\
\hline \multicolumn{2}{c}{$\tilde{p}=2$} \\
\hline $\mathrm{UND}_{\text {trad }}(2,1)$ & 1.9908 \\
$\mathrm{UND}_{3}(2,1)$ & 3.0874 \\
$\mathrm{UND}_{50}(2,1)$ & 1.9962 \\
$\mathrm{UND}_{\mathrm{cc}, 5}(2,2)$ & 2.2480 \\
$\mathrm{UND}_{\mathrm{cc}, 50}(2,2)$ & 3.4308
\end{tabular}

\begin{tabular}{|c|c|c|c|c|c|}
\hline Operator & $\rho_{\text {asym }}$ & Operator & $\rho_{\text {asym }}$ & Operator & $\rho_{\text {asym }}$ \\
\hline \multicolumn{2}{|l|}{$\tilde{p}=4$} & \multicolumn{2}{|c|}{$\tilde{p}=6$} & \multicolumn{2}{|l|}{$\tilde{p}=8$} \\
\hline $\mathrm{UND}_{\text {trad }}(4,2)$ & 2.7435 & $\mathrm{UND}_{\text {trad }}(6,3)$ & 3.1714 & $\mathrm{UND}_{\text {trad }}(8,4)$ & 3.4608 \\
\hline $\mathrm{UND}_{9}(4,2)$ & 2.5202 & $\mathrm{UND}_{13}(6,3)$ & 2.8219 & $\mathrm{UND}_{17}(8,4)$ & 3.0733 \\
\hline $\mathrm{UND}_{50}(4,2)$ & 2.7378 & $\mathrm{UND}_{50}(6,3)$ & 3.1615 & $\mathrm{UND}_{50}(8,4)$ & 3.4463 \\
\hline $\mathrm{UND}_{\mathrm{cc}, 10}(4,4)$ & 2.6093 & $\mathrm{UND}_{\mathrm{cc}, 15}(6,6)$ & 4.0203 & $\mathrm{UND}_{\mathrm{cc}, 60}(8,8)$ & 3.4511 \\
\hline $\mathrm{UND}_{\mathrm{cc}, 50}(4,4)$ & 2.7377 & $\mathrm{UND}_{\mathrm{cc}, 50}(6,6)$ & 3.1613 & $\operatorname{HGT}(8,4)$ & 15.3188 \\
\hline $\operatorname{HGT}(4,2)_{\text {trad }}$ & 2.7435 & $\operatorname{HGT}(6,3)_{\text {trad }}$ & 3.3200 & $\operatorname{HGT}_{17}(8,4)$ & 15.5124 \\
\hline $\mathrm{HGT}_{9}(4,2)$ & 4.0508 & $\operatorname{HGT}_{13}(6,3)$ & 6.2616 & $\operatorname{HGT}_{50}(8,4)$ & 15.3316 \\
\hline $\operatorname{HGT}_{50}(4,2)$ & 2.7382 & $\mathrm{HGT}_{50}(6,3)$ & 3.3143 & $\mathrm{HGT}_{\mathrm{cc}, 22}(8,8)$ & 48.7316 \\
\hline $\operatorname{HGT}_{\mathrm{cc}, 12}(4,4)$ & 9.0483 & $\operatorname{HGT}_{\mathrm{cc}, 17}(6,6)$ & 134.0957 & $\operatorname{HGT}_{\mathrm{cc}, 50}(8,8)$ & 45.5306 \\
\hline $\operatorname{HGT}_{\mathrm{cc}, 50}(4,4)$ & 6.7768 & $\operatorname{HGT}_{\mathrm{cc}, 50}(6,6)$ & 55.6286 & $\operatorname{HGTL}(8,4)_{\text {trad }}$ & 3.4608 \\
\hline $\operatorname{HGTL}(4,2)_{\operatorname{trad}}$ & 2.7435 & $\operatorname{HGTL}(6,3)_{\text {trad }}$ & 3.1714 & $\operatorname{HGTL}_{17}(8,4)$ & 3.2048 \\
\hline $\operatorname{HGTL}_{9}(4,2)$ & 2.5839 & $\operatorname{HGTL}_{13}(6,3)$ & 2.9500 & $\operatorname{HGTL}_{50}(8,4)$ & 3.4475 \\
\hline $\operatorname{HGTL}_{50}(4,2)$ & 2.7381 & $\mathrm{HGTL}_{50}(6,3)$ & 3.1623 & $\operatorname{HGTL}_{\mathrm{cc}, 20}(8,8)$ & 4.5372 \\
\hline $\operatorname{HGTL}_{\mathrm{cc}, 10}(4,4)$ & 2.6626 & $\operatorname{HGTL}_{\mathrm{cc}, 15}(6,6)$ & 3.3197 & $\operatorname{HGTL}_{\mathrm{cc}, 50}(8,8)$ & 4.6416 \\
\hline $\operatorname{HGTL}_{\mathrm{cc}, 50}(4,4)$ & 2.7381 & $\operatorname{HGTL}_{\mathrm{cc}, 50}(6,6)$ & 3.5335 & & \\
\hline
\end{tabular}

Table 3: Spectral radius of the spatial discretization matrix for the periodic two dimensional linear convection equation on a Cartesian grid scaled by average mesh spacing.

In contrast to UND and HGTL, the size of HGT elements has a greater impact on the scaled spectral radii. This behaviour is observed for both the classical and corner corrected HGT operators. Furthermore, the chosen optimization of the corner corrections for the HGT operators has resulted in significantly higher scaled spectral radii than their classical counterparts.

285 Finally, the scaled spectral radii of the corner corrected HGT operators is not monotonically increasing with degree: the degree six operators have a much large scaled spectral radius than the degree eight operators. These results 

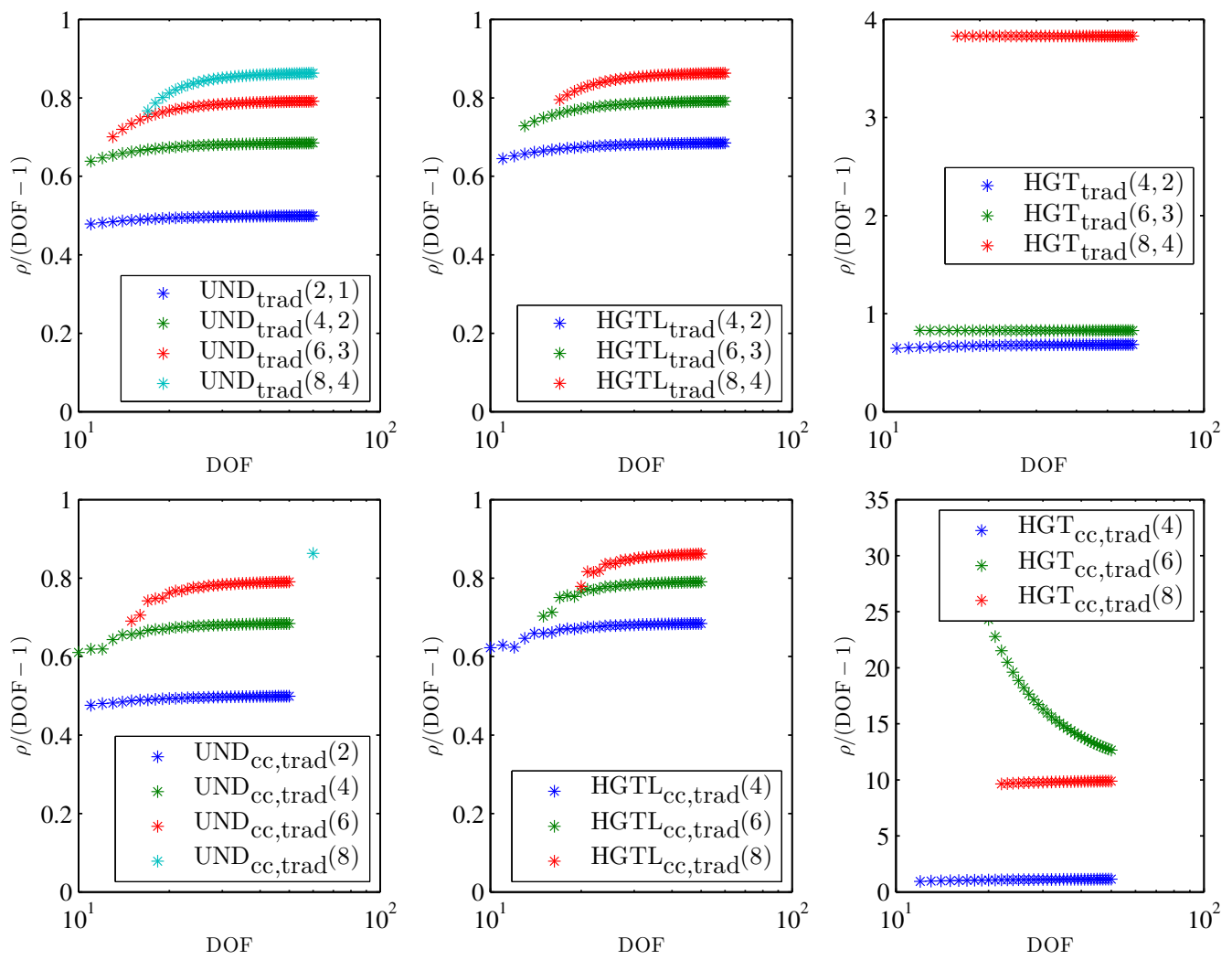

Figure 1: Scaled spectral radii of the spatial discretization matrix as a function of degrees of freedom, DOF, for the one dimensional convection equation.

suggest that alternate optimization strategies should be considered in future work, for example minimum spectral radius or a combination of truncation error and spectral radius.

To further investigate the behavior of the corner-corrected SBP operators as their size increases, Figure 1 shows the scaled spectral radius of classical and corner-corrected operators. These values were computed using the one dimensional linear convection equation with an upwind inflow boundary condition and a single element. Results are shown up to 50 nodes in each operator, with the exception of the degree eight UND corner-corrected operator, which was computed using 60 nodes. The plots show that the asymptotic scaled spectral radii are quickly approached, except for the degree six corner corrected HGT operator which converges from above. Furthermore, the spectral radii of the corner-corrected UND and HGTL operators are very 
similar those of the classical operators with the same interior-point operator and half the global degree. This demonstrates that the corner-corrected operators remain well behaved as the number of nodes in the operator increases, and will, in most cases, have similar maximum stable time steps for explicit time-marching as their classical counterparts of half the global degree.

\subsection{Convergence Rates}

This section presents convergence rates for the proposed corner-corrected SBP operators. These results are generated by convecting the Gaussian initial condition half the width of the domain. The solution is integrated in time using the standard fourth-order Runge-Kutta (RK4) time-marching method. Convergence rates are computed at $t=0.5$ using a fixed time step size of $\Delta t=10^{-5}$. The error is computed with respect to the norm of the discretization, $\mathrm{H}_{\xi}$, as

$$
\|e\|_{\mathrm{H}_{\xi}}=\sqrt{\boldsymbol{e}^{\mathrm{T}} \mathrm{H}_{\xi} \boldsymbol{e}}
$$

where the error vector $\boldsymbol{e}=\boldsymbol{u}_{h}-\boldsymbol{u}$, and $\boldsymbol{u}$ is the exact solution projected onto the grid. The convergence rates are computed between several successively finer meshes,

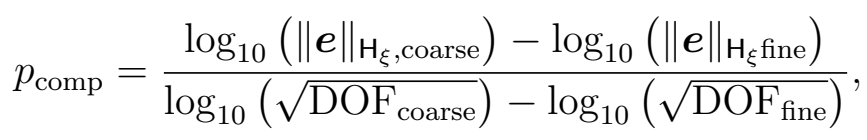

obtained by doubling the number of computational cells in each direction on each level.

Figures 2 and 3 show the convergence of the corner-corrected operators along side their classical counterparts. Results are shown for discretizations using elements with the minimum number of degrees of freedom per element, and 50 degrees of freedom per element. The exception is the $\mathrm{UND}_{\mathrm{cc}, 60}(8)$ which is shown for a discretization using 60 node elements only.

The order of convergence observed for both the classical and cornercorrected operators is one order higher than the global degree of the operator. The corner-corrected operators exhibit this behavior even though the degree of their interior-point operator is not greater than their global degree. This is in contrast to classical operators, where the degree of their interior-point operator is twice the global degree. This behavior is corroborated by the convergence rates tabulated in Appendix A. Furthermore, it is not entirely unexpected; for example, collocated nodal discontinuous Galerkin approaches 

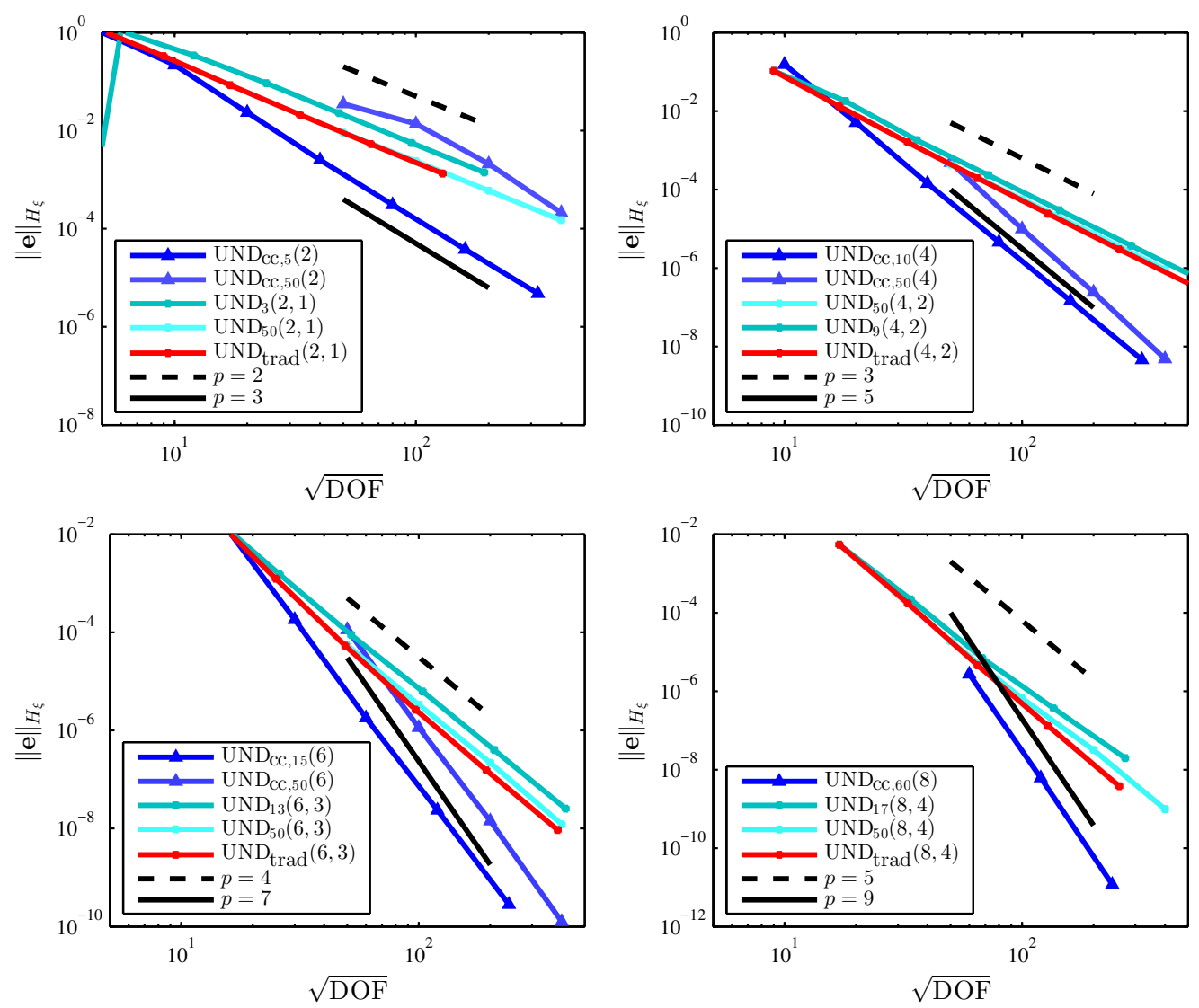

Figure 2: Convergence plots for UND operators with and without corner corrections for interior-point operators of degree $\tilde{p}=2,4,6,8$.

that use operators of uniform degree, such as Legendre-Gauss-Lobatto, also exhibit $p+1$ convergence when using upwind flux functions [30]. The $p+1$ convergence rate of the corner-corrected operators continues as the number of interior-point operators increases and the corner corrections become less and less local. The error of the corner-corrected operators does increase with the number of repeating interior-point operators; however, the growth is not catastrophic. Therefore, the corner corrections can be applied to existing classical SBP simulations with moderate element sizes and be expected to give reasonable results. 

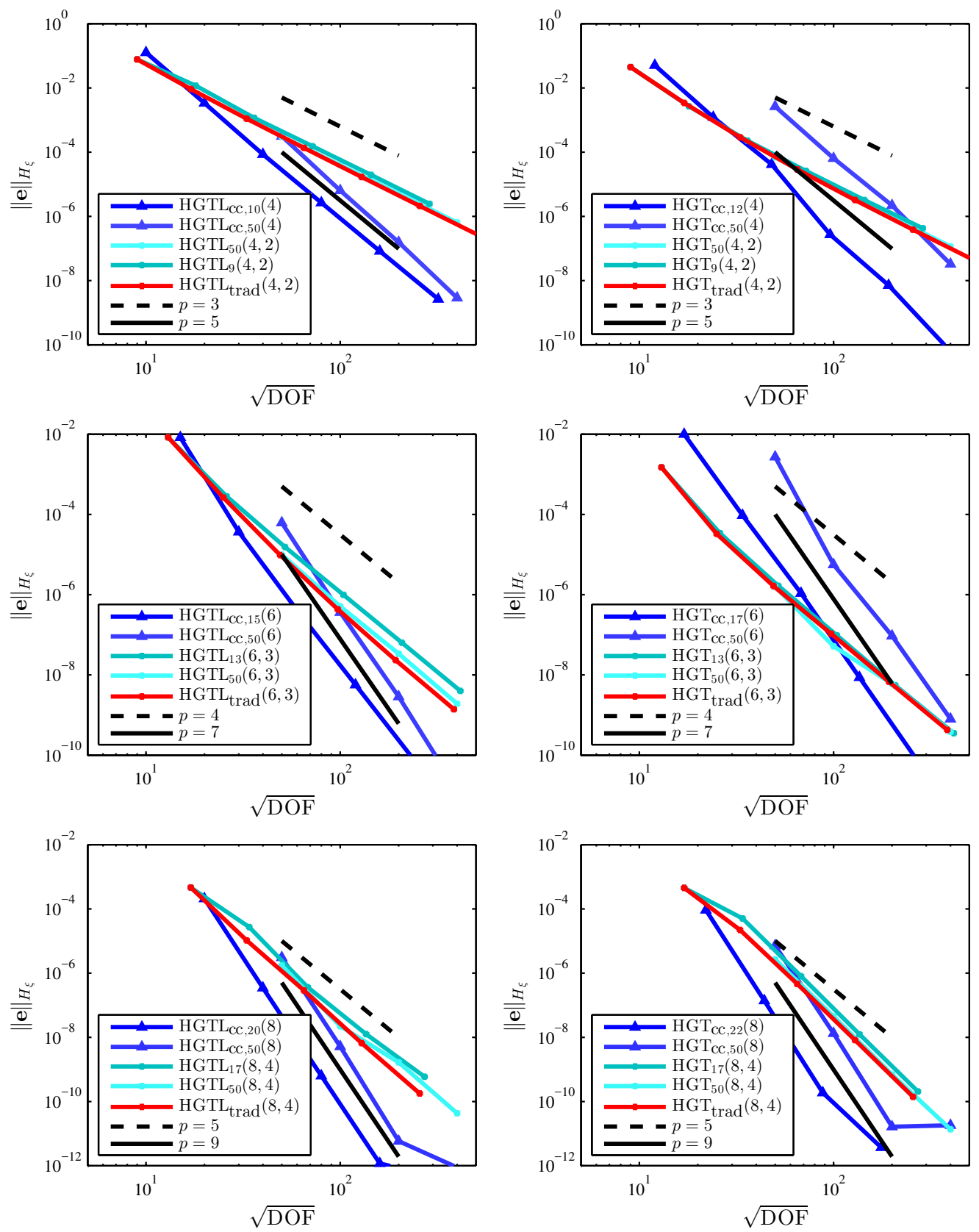

Figure 3: Convergence plots for HTGL (left) and HGT (right) operators with and without corner corrections for interior-point operators of degree $\tilde{p}=4,6,8$. 


\subsection{Relative Efficiency}

In this section, the efficiency of the proposed operators is investigated relative to their classical counterparts. The solution is integrated in time using the standard fourth-order Runge-Kutta (RK4) time-marching method to $t=3$, corresponding to three passes of the Gaussian through the domain. In this case, the time step size is computed as $\Delta t=\frac{\overline{\Delta x}}{10 \rho_{\text {asym }}}$, where $\rho_{\text {asym }}$ is the asymptotic value of the spectral radius of the spatial discretization matrix scaled by the average mesh spacing, $\overline{\Delta x}$. The scaled spectral radius is precomputed for each operator, as previously discussed. This analysis combines the influence of both relative accuracy and spectral radius. Figures 4 and 5 summarize these results, organized by the degree of the interior point operator $\tilde{p}$.

The relative efficiency of the classical and corner-corrected operators is dependent on the desired level of accuracy. The classical operators tend to be more efficient for simulations requiring less stringent accuracy, while the higher-order corner-corrected operators become more efficient as the desired accuracy becomes more stringent. This type of crossover is common when comparing operators of different degree, and the location of the crossover point is problem dependent. In cases requiring high accuracy, the cornercorrected operators present a higher order and more efficient alternative. In addition, the higher-order corner corrected discretizations maintain the same repeating interior-point operator as the classical operators and similar spectral radii.

These Figures also show the importance of spectral radius in practical computation. While the corner corrected HGT operators have competitive accuracy as a function of degrees of freedom (See Section 6.3), their large spectral radius (See Section 6.2) render them the least efficient of the corner corrected operators. This further motivates the consideration of different objective functions in the optimization of the corner corrections, proposed for future work. 

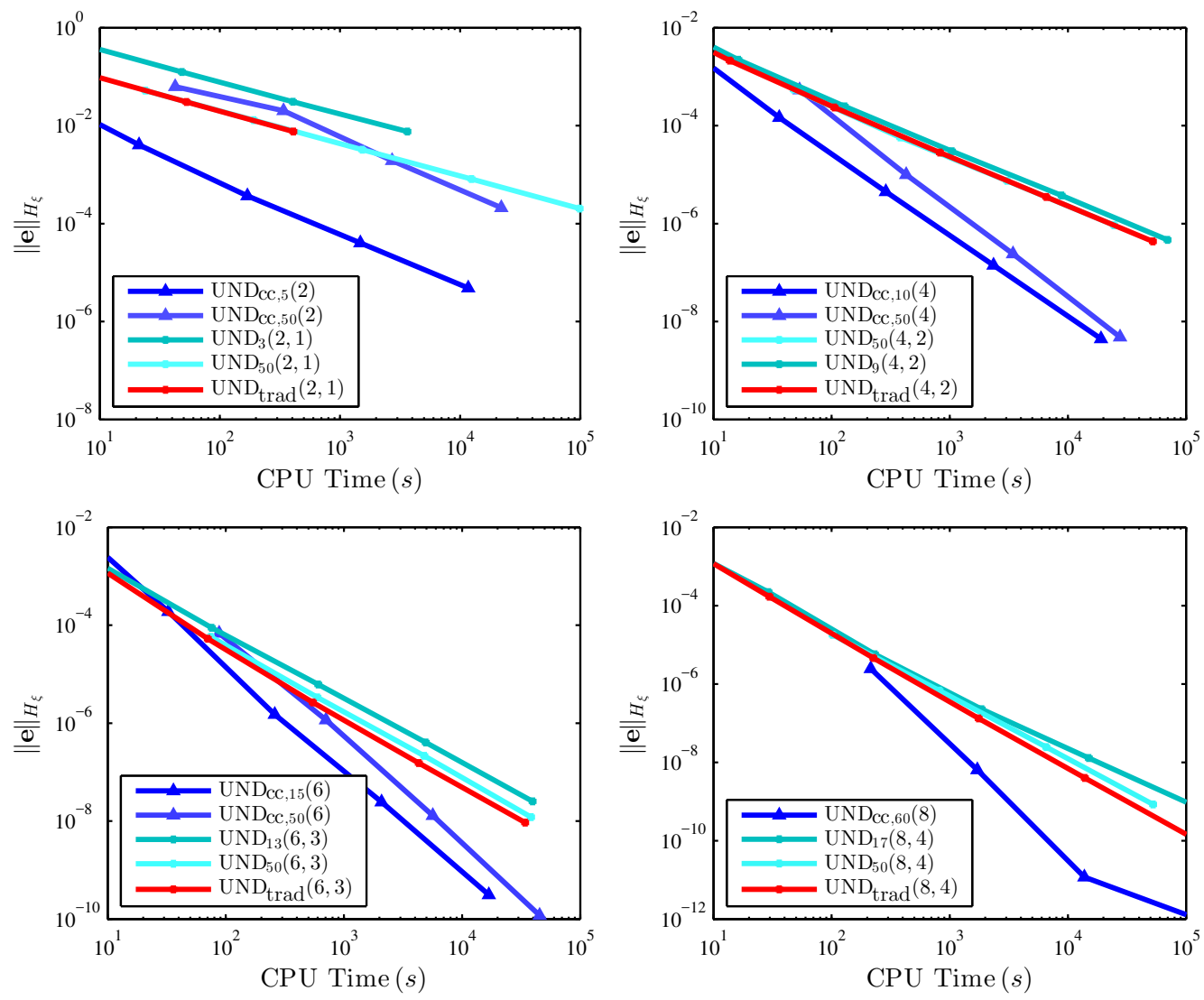

Figure 4: Efficiency plots for UND operators using a constant factor of the maximum stable time step. 

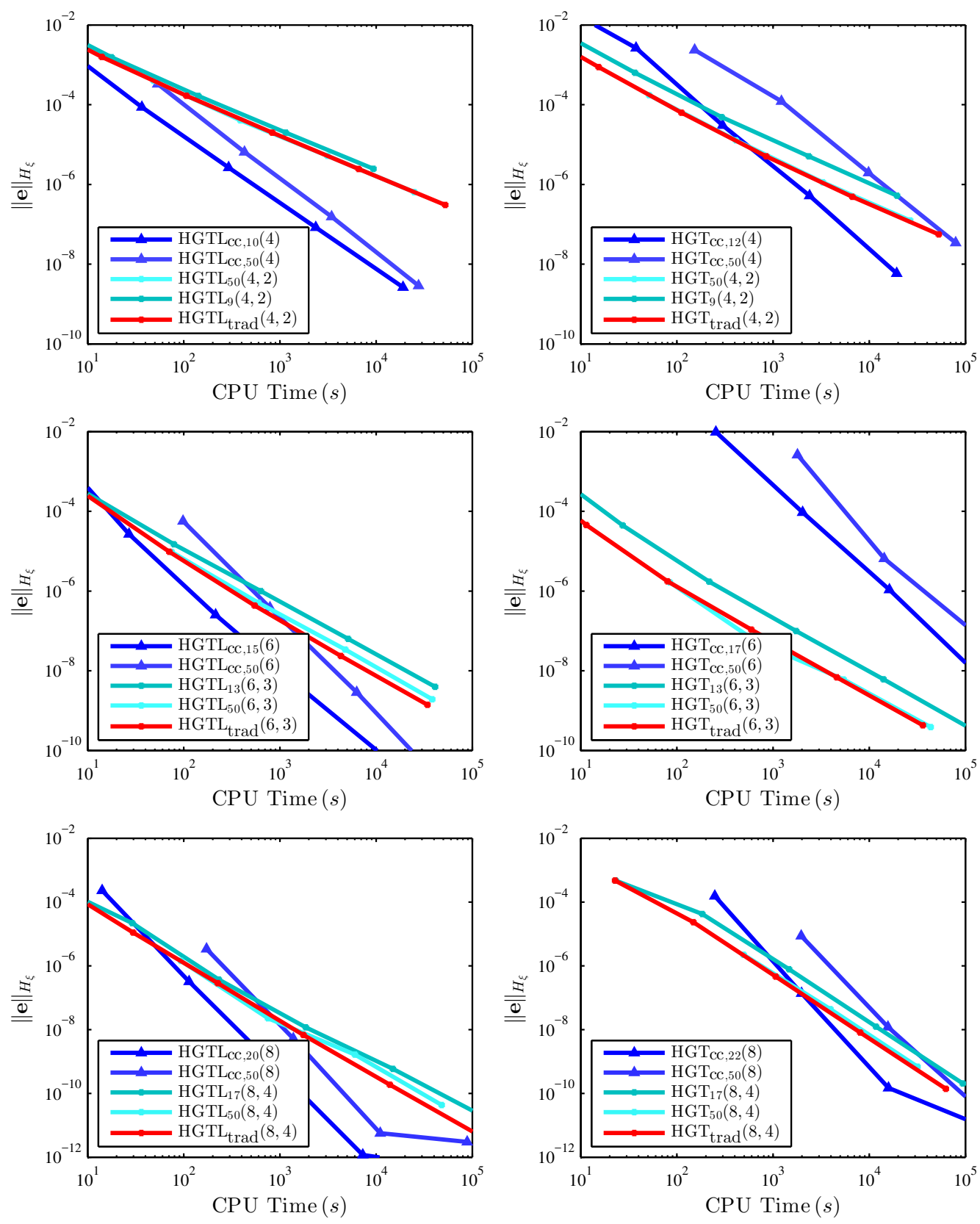

Figure 5: Efficiency plots for HGTL (left) and HGT (right) operators using a constant factor of the maximum stable time step. 


\section{Conclusions}

We modify the structure of FD-SBP operators to enable the construction of operators with a repeating interior-point operator that are of degree $2 p$ at all nodes and develop an approach to find general solutions for this class of operators.

The main conclusions are:

- In almost all cases, the corner-corrected operators had solution error of order $p+1$

- They have comparable spectral radii of the spatial discretization matrix to SBP operators with the same repeating interior-point operator yet they have almost twice the order for the solution error

- As the number of nodes is increased, the truncation error increases; however, the operators remain well-behaved for all element sizes investigated here, i.e., up to 50 nodes

The results in this paper show that for the same interior-point operator we obtain schemes with nearly twice the order of accuracy while maintaining the same characteristics of classical SBP methods. Moreover, the UND cornercorrected operators offer an immediate means of extending existing SBP codes using classical SBP operators to up to global degree 8 with well behaved operators.

Our future work will concentrate on improving these operators by introducing additional degrees of freedom, which have previously been shown to lead to more efficient operators [11], and optimizing the operators for spectral radius in addition to their error characteristics. 


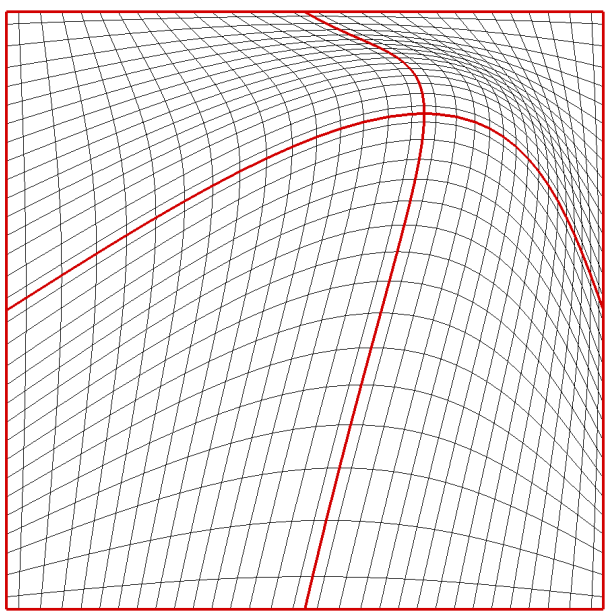

Figure A.6: Four-element curvilinear mesh using $17 \times 17$ node $\operatorname{HGTL}(8,4)$ nodal distribution.

\section{Appendix A. Curvilinear coordinates}

To demonstrate the results presented in this paper hold in curvilinear coordinates, the convergence simulations presented in Section 6.3 are repeated using the following transformation

$$
\begin{aligned}
& x=\xi+\frac{1}{5} \sin (\pi \xi) \sin (\pi \eta) \\
& y=\eta+\frac{1}{5} \exp (1-\eta) \sin (\pi \xi) \sin (\pi \eta)
\end{aligned}
$$

applied to the Cartesian decomposition described in Section 6.1. The transformation in physical space is shown in Figure A.6 for a uniform nodal distribution. The simulation results are summarized in Tables A.4 through A.13. Results for HGT operators on curved meshes have been omitted, as stable and conservative SATs on curved meshes for operators not including both boundary nodes is an ongoing area of research.

The convergence rate in each case is greater than global degree $p$, and in most cases one order higher than global degree. The most significant deviation from this behavior is the degree six corner corrected simulations. These converge at a rate greater than the global degree $p$, but only about a quarter of an order higher, rather an a full order higher. 


\begin{tabular}{lc|c|cc}
$\begin{array}{l}\text { Cartesian with Upwind SATs } \\
\log (e)\end{array}$ & $p_{\text {comp }}$ & $N$ & \multicolumn{2}{c}{ Curved with Upwind SATs } \\
\multicolumn{5}{c}{$\mathrm{UND}_{\text {trad }}(2,1)$} \\
\hline 0.04 & 25 & 0.04 & $p_{\text {comp }}$ \\
-0.48 & -2.04 & 81 & -0.28 & -1.24 \\
-1.07 & -2.14 & 289 & -0.80 & -1.89 \\
-1.67 & -2.08 & 1089 & -1.36 & -1.94 \\
-2.27 & -2.04 & 4225 & -1.96 & -2.04 \\
-2.88 & -2.02 & 16641 & -2.56 & -2.03 \\
\hline \multicolumn{5}{c}{$\mathrm{UND}_{\mathrm{cc}, 5}(2)$} \\
\hline-0.66 & -100 & -0.37 \\
-1.63 & -3.22 & 400 & -0.98 \\
-2.60 & -3.21 & 1600 & -1.81 & -2.03 \\
-3.51 & -3.05 & 6400 & -2.84 & -2.77 \\
-4.42 & -3.01 & 25600 & -3.79 & -3.42 \\
-5.32 & -3.00 & 102400 & -4.69 & -2.98
\end{tabular}

Table A.4: Convergence rates for UND operators with interior-point operators of degree $\tilde{p}=2$.

\begin{tabular}{lc|c|cc}
$\begin{array}{l}\text { Cartesian with Upwind SATs } \\
\log (e)\end{array}$ & $p_{\text {comp }}$ & $N$ & \multicolumn{2}{|c}{ Curved with Upwind SATs } \\
\multicolumn{5}{c}{$\mathrm{UND}_{\text {trad }}(4,2)$} \\
\hline-1.88 & 289 & -1.42 & $p_{\text {comp }}$ \\
-2.80 & -3.21 & 1089 & -2.30 & -3.04 \\
-3.71 & -3.10 & 4225 & -3.33 & -3.50 \\
-4.62 & -3.05 & 16641 & -4.37 & -3.50 \\
-5.52 & -3.02 & 66049 & -5.40 & -3.43 \\
-6.43 & -3.01 & 263169 & -6.41 & -3.36 \\
\hline \multicolumn{5}{c}{} \\
\hline-2.29 & $-\mathrm{UND}_{\mathrm{cc}, 10}(4)$ \\
-3.85 & -5.16 & 400 & -1.14 \\
-5.34 & -4.97 & 1600 & -2.23 \\
-6.84 & -4.97 & 6400 & -3.86 & -3.62 \\
-8.34 & -4.98 & 25600 & -5.38 \\
\end{tabular}

Table A.5: Convergence rates for UND operators with interior-point operators of degree $\tilde{p}=4$. 


\begin{tabular}{|c|c|c|c|c|}
\hline \multicolumn{3}{|c|}{ Cartesian with Upwind SATs } & \multicolumn{2}{|c|}{ Curved with Upwind SATs } \\
\hline $\log (e)$ & $p_{\text {comp }}$ & $N$ & $\log (e)$ & $p_{\text {comp }}$ \\
\hline \multicolumn{5}{|c|}{$\mathrm{HGTL}_{\text {trad }}(4,2)$} \\
\hline-2.04 & & 289 & -1.50 & \\
\hline-2.96 & -3.19 & 1089 & -2.43 & -3.22 \\
\hline-3.87 & -3.09 & 4225 & -3.48 & -3.58 \\
\hline-4.77 & -3.04 & 16641 & -4.54 & -3.55 \\
\hline-5.68 & -3.02 & 66049 & -5.57 & -3.46 \\
\hline-6.58 & -3.01 & 263169 & -6.58 & -3.36 \\
\hline \multicolumn{5}{|c|}{$\operatorname{HGTL}_{9}(4,2)$} \\
\hline-1.93 & & 324 & -1.48 & \\
\hline-2.93 & -3.33 & 1296 & -2.32 & -2.77 \\
\hline-3.82 & -2.93 & 5184 & -3.22 & -2.99 \\
\hline-4.71 & -2.97 & 20736 & -4.17 & -3.16 \\
\hline-5.61 & -3.00 & 82944 & -5.02 & -2.82 \\
\hline \multicolumn{5}{|c|}{$\operatorname{HGTL}_{\mathrm{cc}, 10}(4)$} \\
\hline-2.47 & & 400 & -1.22 & \\
\hline-4.07 & -5.32 & 1600 & -2.36 & -3.79 \\
\hline-5.58 & -5.00 & 6400 & -4.08 & -5.70 \\
\hline-7.08 & -5.00 & 25600 & -5.60 & -5.06 \\
\hline-8.58 & -4.98 & 102400 & -7.11 & -5.03 \\
\hline
\end{tabular}

Table A.6: Convergence rates for HGTL operators with interior-point operators of degree $\tilde{p}=4$. 
Curved with Upwind SATs

\begin{tabular}{c|lc}
$N$ & $\log (e)$ & $p_{\text {comp }}$ \\
\hline \multicolumn{3}{c}{$\operatorname{HGT}_{9}(4,2)$} \\
\hline 81 & -0.83 \\
324 & -2.02 & -3.94 \\
1296 & -3.20 & -3.94 \\
5184 & -4.31 & -3.70 \\
20736 & -5.30 & -3.26 \\
82944 & -6.29 & -3.29 \\
\hline \multicolumn{3}{c}{ HGT $_{\text {trad }}(4,2)$} \\
\hline 81 & -0.83 \\
289 & -1.92 & -3.93 \\
1089 & -3.06 & -3.97 \\
4225 & -4.20 & -3.89 \\
16641 & -5.30 & -3.68 \\
66049 & -6.31 & -3.38 \\
263169 & -7.25 & -3.15 \\
\hline \multicolumn{3}{c}{$\mathrm{HGT}_{\mathrm{cc}, 12}(4)$} \\
\hline 144 & -1.36 & \\
576 & -2.58 & -4.06 \\
2304 & -4.52 & -6.44 \\
9216 & -6.29 & -5.88 \\
36864 & -8.23 & -6.46
\end{tabular}

Table A.7: Convergence rates for HGT operators with interior-point operators of degree $\tilde{p}=4$. 


\begin{tabular}{lc|c|ll}
$\begin{array}{l}\text { Cartesian with Upwind SATs } \\
\log (e)\end{array}$ & $p_{\text {comp }}$ & $N$ & \multicolumn{2}{|c}{ Curved with Upwind SATs } \\
\hline \multicolumn{5}{c}{$\mathrm{UND}_{\text {trad }}(6,3)$} \\
\hline-2.91 & 625 & -1.82 & $p_{\text {comp }}$ \\
-4.27 & -4.68 & 2401 & -3.08 & -4.31 \\
-5.58 & -4.39 & 9409 & -4.40 & -4.45 \\
-6.82 & -4.15 & 37249 & -5.81 & -4.73 \\
-8.03 & -4.05 & 148225 & -7.24 & -4.76 \\
\hline \multicolumn{5}{c}{$\mathrm{UND}_{\text {cc, }, 15}(6)$} \\
\hline-3.74 & -600 & -2.03 \\
-5.74 & -6.65 & 9000 & -3.54 \\
-7.63 & -6.39 & 14400 & -5.40 \\
-9.55 & 57600 & -7.29 & -6.04 \\
\hline
\end{tabular}

Table A.8: Convergence rates for UND operators with interior-point operators of degree $\tilde{p}=6$. 


\begin{tabular}{|c|c|c|c|c|}
\hline \multicolumn{3}{|c|}{ Cartesian with Upwind SATs } & \multicolumn{2}{|c|}{ Curved with Upwind SATs } \\
\hline $\log (e)$ & $p_{\text {comp }}$ & $N$ & $\log (e)$ & $p_{\text {comp }}$ \\
\hline \multicolumn{5}{|c|}{$\mathrm{HGTL}_{\text {trad }}(6,3)$} \\
\hline-3.58 & & 625 & -2.32 & \\
\hline-5.02 & -4.91 & 2401 & -3.69 & -4.67 \\
\hline-6.36 & -4.54 & 9409 & -5.08 & -4.68 \\
\hline-7.63 & -4.24 & 37249 & -6.51 & -4.81 \\
\hline-8.85 & -4.08 & 148225 & -7.94 & -4.75 \\
\hline \multicolumn{5}{|c|}{$\mathrm{HGTL}_{13}(6,3)$} \\
\hline-3.55 & & 676 & -2.33 & \\
\hline-4.81 & -4.19 & 2704 & -3.60 & -4.22 \\
\hline-6.01 & -3.96 & 10816 & -5.05 & -4.83 \\
\hline-7.20 & -3.96 & 43264 & -6.12 & -3.57 \\
\hline-8.40 & -3.99 & 173056 & & \\
\hline \multicolumn{5}{|c|}{$\mathrm{HGTL}_{\mathrm{cc}, 15}(6)$} \\
\hline-4.44 & & 900 & -2.25 & \\
\hline-6.39 & -6.48 & 3600 & -4.00 & -5.81 \\
\hline-8.24 & -6.17 & 14400 & -6.03 & -6.75 \\
\hline-10.11 & -6.21 & 57600 & -7.90 & -6.21 \\
\hline
\end{tabular}

Table A.9: Convergence rates for HGTL operators with interior-point operators of degree $\tilde{p}=6$. 
Cartesian with Upwind SATs

\begin{tabular}{c|ll}
$N$ & $\log (e)$ & $p_{\text {comp }}$ \\
\hline \multicolumn{3}{|c}{$\operatorname{HGT}_{13}(6,3)$} \\
\hline 169 & -2.73 \\
676 & -4.35 & -5.39 \\
2704 & -5.77 & -4.70 \\
10816 & -7.00 & -4.11 \\
43264 & -8.21 & -4.02 \\
173056 & -9.45 & -4.11 \\
\hline \multicolumn{3}{|c}{$\mathrm{HGT}_{\text {trad }}(6,3)$} \\
\hline 169 & -2.73 \\
625 & -4.35 & -5.69 \\
2401 & -5.76 & -4.83 \\
9409 & -6.97 & -4.08 \\
37249 & -8.17 & -4.02 \\
148225 & -9.37 & -4.01 \\
\hline \multicolumn{3}{|c}{$\mathrm{HGT}_{\mathrm{cc}, 17}(6)$} \\
\hline 289 & \multicolumn{2}{|l}{-2.01} \\
1156 & -4.03 & -6.70 \\
4624 & -5.97 & -6.44 \\
18496 & -8.06 & -6.96
\end{tabular}

Table A.10: Convergence rates for HGT operators with interior-point operators of degree $\tilde{p}=6$. 


\begin{tabular}{lc|c|ll}
$\begin{array}{l}\text { Cartesian with Upwind SATs } \\
\log (e)\end{array}$ & $p_{\text {comp }}$ & $N$ & \multicolumn{2}{|c}{ Curved with Upwind SATs } \\
\hline \multicolumn{5}{c}{$\mathrm{UND}_{\text {trad }}(8,4)$} \\
\hline-3.76 & 1089 & -2.47 & $p_{\text {comp }}$ \\
-5.34 & -5.36 & 4225 & -4.03 & -5.29 \\
-6.89 & -5.20 & 16641 & -5.47 & -4.84 \\
-8.42 & -5.12 & 66049 & -6.94 \\
\hline \multicolumn{5}{c}{-4.90} \\
\hline-8.21 & $\mathrm{UND}_{\mathrm{cc}, 60}(8)$ \\
-10.93 & 14400 & -5.35 \\
\end{tabular}

Table A.11: Convergence rates for UND operators with interior-point operators of degree $\tilde{p}=8$.

\begin{tabular}{|c|c|c|c|c|}
\hline \multicolumn{3}{|c|}{ Cartesian with Upwind SATs } & \multicolumn{2}{|c|}{ Curved with Upwind SATs } \\
\hline $\log (e)$ & $p_{\text {comp }}$ & $N$ & $\log (e)$ & $p_{\text {comp }}$ \\
\hline \multicolumn{5}{|c|}{$\operatorname{HGTL}_{\text {trad }}(8,4)$} \\
\hline-4.98 & & 1089 & -3.01 & \\
\hline-6.54 & -5.30 & 4225 & -4.98 & -6.71 \\
\hline-8.18 & -5.50 & 16641 & -6.59 & -5.40 \\
\hline-9.75 & -5.24 & 66049 & -8.04 & -4.86 \\
\hline-9.65 & -5.37 & 263169 & & \\
\hline \multicolumn{5}{|c|}{$\operatorname{HGTL}_{17}(8,4)$} \\
\hline-4.57 & & 1156 & -3.01 & \\
\hline-6.44 & -6.22 & 4624 & -4.84 & -6.11 \\
\hline-7.90 & -4.84 & 18496 & -6.24 & -4.64 \\
\hline-9.23 & -4.42 & 73984 & -7.97 & -5.75 \\
\hline \multicolumn{5}{|c|}{$\operatorname{HGTL}_{\mathrm{cc}, 20}(8)$} \\
\hline-6.46 & & 1600 & -3.35 & \\
\hline-9.20 & -9.10 & 6400 & -5.71 & -7.81 \\
\hline-11.93 & -9.06 & 25600 & -8.10 & -7.97 \\
\hline-12.35 & -1.39 & 102400 & -10.95 & -9.46 \\
\hline
\end{tabular}

Table A.12: Convergence rates for HGTL operators with interior-point operators of degree $\tilde{p}=8$. 
Cartesian with Upwind SATs

\begin{tabular}{|c|c|c|}
\hline$N$ & $\log (e)$ & $p_{\text {comp }}$ \\
\hline \multicolumn{3}{|c|}{$\operatorname{HGT}_{17}(8,4)$} \\
\hline 289 & -3.33 & \\
\hline 1156 & -4.38 & -3.48 \\
\hline 4624 & -6.11 & -5.75 \\
\hline 18496 & -7.91 & -5.98 \\
\hline 73984 & -9.69 & -5.92 \\
\hline \multicolumn{3}{|c|}{$\operatorname{HGT}_{\text {trad }}(8,4)$} \\
\hline 289 & -3.33 & \\
\hline 1089 & -4.63 & -4.53 \\
\hline 4225 & -6.34 & -5.78 \\
\hline 16641 & -8.09 & -5.90 \\
\hline 66049 & -9.86 & -5.90 \\
\hline \multicolumn{3}{|c|}{$\operatorname{HGT}_{\mathrm{cc}, 22}(8)$} \\
\hline 484 & -3.82 & \\
\hline 1936 & -6.86 & -10.13 \\
\hline 7744 & -9.83 & -9.84 \\
\hline 30976 & -10.93 & -3.68 \\
\hline
\end{tabular}

Table A.13: Convergence rates for HGT operators with interior-point operators of degree $\tilde{p}=8$. 


\section{Appendix B. Matlab ${ }^{\circledR}$ code}

Here we list the various Matlab ${ }^{\complement}$ files and their functions:

- Run_convergence.m: Driving routine to run a convergence study for a

400

405 specific operator

- convergence.m: Subroutine that runs a convergence study

- construct_spatial_LC_trad.m: Constructs the spatial discretization using the traditional finite-difference approach

- construct_spatial_LC.m: Constructs the spatial discretization using the discontinuous element approach

- construct_spatial_LC_continuous.m: Constructs the spatial discretization using the continuous element approach

- operator.m: returns the SBP operator in computational space

The SBP operators are constructed using the functions

- UND: UNDpEQ1.m, UNDpEQ2.m, UNDpEQ3.m UNDpEQ4.m, UNDpEQ1DegEQ2.m UNDpEQ2DegEQ3.m, UNDpEQ2DegEQ4.m, UNDpEQ3DegEQ5.m, UNDpEQ3DegEQ6.m

- HGTL: HGTLpEQ2.m, HGTLpEQ3.m, HGTLpEQ4.m, HGTLpEQ2DegEQ4.m, HGTLpEQ3DegEQ6.m, HGTLpEQ4DegEQ8.m

- HGT: HGTpEQ2.m, HGTpEQ3.m, HGTpEQ4.m, HGTpEQ2DegEQ4.m, HGTpEQ3DegEQ6.m, HGTpEQ4DegEQ8.m

\section{References}

\section{References}

[1] D. C. Del Rey Fernández, J. E. Hicken, D. W. Zingg, Review of summation-by-parts operators with simultaneous approximation terms for the numerical solution of partial differential equations, Computers \& Fluids 95 (22) (2014) 171-196. 
[2] M. Svärd, J. Nordström, Review of summation-by-parts schemes for initial-boundary-value-problems, Journal of Computational Physics 268 (1) (2014) 17-38.

[3] D. C. Del Rey Fernández, P. D. Boom, D. W. Zingg, A generalized framework for nodal first derivative summation-by-parts operators, Journal of Computational Physics 266 (1) (2014) 214-239.

[4] G. J. Gassner, A skew-symmetric discontinuous Galerkin spectral element discretization and its relation to SBP-SAT finite difference methods, SIAM Journal on Scientific Computing 35 (3) (2013) A1233-A1253.

[5] M. H. Carpenter, D. Gottlieb, Spectral methods on arbitrary grids, Journal of Computational Physics 129 (1) (1996) 74-86.

[6] M. H. Carpenter, D. Gottlieb, S. Abarbanel, Time-stable boundary conditions for finite-difference schemes solving hyperbolic systems: Methodology and application to high-order compact schemes, Journal of Computational Physics 111 (2) (1994) 220-236.

[7] M. H. Carpenter, J. Nordström, D. Gottlieb, A stable and conservative interface treatment of arbitrary spatial accuracy, Journal of Computational Physics 148 (2) (1999) 341-365.

[8] J. Nordström, M. H. Carpenter, Boundary and interface conditions for high-order finite-difference methods applied to the Euler and NavierStokes equations, Journal of Computational Physics 148 (2) (1999) 621645 .

[9] J. Nordström, M. H. Carpenter, High-order finite-difference methods, multidimensional linear problems, and curvilinear coordinates, Journal of Computational Physics 173 (1) (2001) 149-174.

[10] J. E. Hicken, D. C. Del Rey Fernández, D. W. Zingg, Multi-dimensional summation-by-parts operators: General theory and application to simplex elements, SIAM Journal on Scientific Computing 38 (4) (2016) A1935-A1958.

[11] K. Mattsson, M. Almquist, M. H. Carpenter, Optimal diagonal-norm SBP operators, Journal of Computational Physics 264 (1) (2014) 91111. 
[12] D. C. Del Rey Fernández, D. W. Zingg, Generalized summation-by-parts operators for the second derivative with a variable coefficient, SIAM Journal on Scientific Computing 37 (6) (2015) A2840-A2864.

[13] N. Albin, J. Klarmann, An algorithmic exploration of the existence of high-order summation by parts operators with diagonal norm, Journal of Scientific Computing (2016) 1-18.

[14] P. Diener, E. N. Dorband, E. Schnetter, M. Tiglio, Optimized high-order derivative and dissipation operators satisfying summation by parts, and applications in three-dimensional multi-block evolutions, Journal of Scientific Computing 32 (1) (2007) 109-145.

[15] J. E. Hicken, D. W. Zingg, Superconvergent functional estimates from summation-by-parts finite-difference discretizations, SIAM Journal on Scientific Computing 33 (2) (2011) 893-922.

[16] K. Mattsson, Summation by parts operators for finite difference approximations of second-derivatives with variable coefficients, Journal of Scientific Computing 51 (3) (2012) 650-682.

[17] K. Mattsson, J. Nordström, Summation by parts operators for finite difference approximations of second derivatives, Journal of Computational Physics 199 (2004) 503-540.

[18] B. Gustafsson, H.-O. Kreiss, J. Oliger, Time-Dependent Problems and Difference Methods, 2nd Edition, Pure and Applied Mathematics, Wiley, 2013.

[19] B. Gustafsson, High Order Difference Methods for Time Dependent PDE, Springer, 2008.

[20] J. E. Hicken, D. W. Zingg, Summation-by-parts operators and highorder quadrature, Journal of Computational and Applied Mathematics 237 (1) (2013) 111-125.

[21] M. Svärd, On coordinate transformations for summation-by-parts operators, Journal of Scientific Computing 20 (1) (2004) 29-42.

[22] J. E. Hicken, D. C. Del Rey Fernández, D. W. Zingg, Opportunities for efficient high-order methods based on the summation-by-parts property, 
22nd AIAA Computational Fluid Dynamics Conference, AIAA Paper 2015-3198, Dallas, Texas, USA, June2015.

[23] B. K. Alpert, Hybrid Gauss-trapezoidal quadrature rules, SIAM Journal on Scientific Computing 5 (1999) 1551-1584.

[24] K. Mattsson, M. H. Carpenter, Stable and accurate interpolation operators for high-order multiblock finite difference methods, SIAM Journal on Scientific Computing 32 (4) (2010) 2298-2320.

[25] D. C. Del Rey Fernández, D. W. Zingg, New diagonal-norm summationby-parts operators for the first derivative with increased order of accuracy, AIAA Aviation 2015, Paper 2015-2914.

[26] M. Parsani, M. H. Carpenter, E. J. Nielsen, Entropy stable wall boundary conditions for the three-dimensional compressible navier-stokes equations, Journal of Computational Physics 292 (C) (2015) 88-113.

[27] M. H. Carpenter, T. C. Fisher, E. J. Nielsen, S. H. Frankel, Entropy stable spectral collocation schemes for the navier-stokes equations: Discontinuous interfaces, SIAM Journal on Scientific Computing 36 (5) (2014) B835B867.

[28] T. C. Fisher, M. H. Carpenter, High-order entropy stable finite difference schemes for nonlinear conservation laws: Finite domains, Journal of Computational Physics 252 (1) (2013) 518-557.

[29] G. J. Gassner, A. R. Winters, D. A. Kopriva, Split form nodal discontinuous Galerkin schemes with summation-by-parts property for the compressible Euler equations, arXiv:1604.06618v2 [math.NA] (2016) 28-50.

[30] J. S. Hesthaven, T. Warburton, Nodal Discontinuous Galerkin Methods Algorithms, Analysis, and Applications, Springer, 2008.

[31] K. Mattsson, M. Svärd, M. Shoeybi, Stable and accurate schemes for the compressible Navier-Stokes equations, Journal of Computational Physics 227 (4) (2008) 2293-2316.

[32] M. Svärd, J. Nordström, A stable high-order finite difference scheme 515 for the compressible Navier-Stokes equations: No-slip wall boundary conditions, Journal of Computational Physics 227 (10) (2008) 48054824 . 
[33] M. Svärd, M. H. Carpenter, J. Nordström, A stable high-order finite difference scheme for the compressible Navier-Stokes equations, far-field boundary conditions, Journal of Computational Physics 225 (1) (2007) 1020-1038. 